\title{
A mathematical model for the transmission of Louse-Borne Relapsing Fever
}

\author{
Ahuod Alsheri and Stephen A. Gourley* \\ *Department of Mathematics, University of Surrey, Guildford, Surrey, GU2 7XH, UK.
}

\begin{abstract}
We present a detailed derivation and analysis of a model consisting of seven coupled delay differential equations for Louse Borne Relapsing Fever (LBRF), a disease transmitted from human to human by the body louse Pediculus humanus humanus. Delays model the latency stages of LBRF in humans and lice, which vary in duration from individual to individual, and are therefore modelled using distributed delays with relatively general kernels. A particular feature of the transmission of LBRF to a human is that it involves the death of the louse, usually by crushing which has the effect of releasing the infected body fluids of the dead louse onto the hosts skin. Careful attention is paid to this aspect. We obtain results on existence, positivity, boundedness, linear and nonlinear stability, and persistence. We also derive a basic reproduction number $R_{0}$ for the model and discuss its dependence on the model parameters. Our analysis of the model suggests that effective louse control without crushing should be the best strategy for LBRF eradication. We conclude that simple measures and precautions should, in general, be sufficient to facilitate disease eradication.
\end{abstract}

Key words: Louse Borne Relapsing Fever; Vector-Borne Disease; Epidemic; Delay; Stability; Basic Reproduction Number.

Short title: Model of Louse-Borne Relapsing Fever

\section{Introduction}

Infectious diseases associated with human lice can be lethal if not promptly or effectively treated. One such disease is Louse-Borne Relapsing Fever (LBRF) which is caused by a spirochaete bacteria called Borrelia recurrentis, transmitted by the body louse Pediculus humanus humanus. The bacteria $B$. recurrentis is related to $B$. duttonii, which causes another type of relapsing fever known as Tick-Borne Relapsing Fever (TBRF). In this paper we present a detailed derivation and analysis of a mathematical model for LBRF.

In the absence of proper treatment, mortality due to LBRF can vary from $10 \%-40 \%$, decreasing with treatment to $2 \%-5 \%$ (Raoult et al [14], Cutler et al [3]). Patients experience episodes of fever, headache, muscle and joint aches and nausea and, in the absence of treatment, illness can be severe (Badiaga et al [1], Southern and Sanford [16]). After a first remission the spirochaetes reappear in the blood causing a relapse, giving rise to the name of the disease. Without treatment there may be several relapses, but LBRF can be treated using antibiotics [1]. Due to antigenic variation of Borrelia strains, 
infection confers a partial immunity. The relapsing phenomenon is not within the scope of the present paper since it requires detailed modelling of the immune response within a human host and the fact that $B$. recurrentis has evolved effective immune evasion strategies (Meri et al [12]).

In the first half of the last century, LBRF reached epidemic levels after World Wars I and II, especially in refugee camps in eastern Europe, the former Soviet Union and Africa. In more recent times there have been a number of LBRF outbreaks in Ethiopia, Sudan, Burundi, Rwanda and the Democratic Republic of the Congo (Raoult et al [14], Hoch et al [8], Ciervo et al [2]). Historically the disease was common in places involving refugees, asylum seekers, homeless people, prisoners and slum dwellers. Outbreaks tend to be exacerbated by the combination of cold weather and lack of hygiene. This can be explained by the fact that people are likely to be wearing more clothing in such conditions and, if this is shared and unwashed, conditions become very suitable for the spread of body lice and the body fluids of dead lice, and the diseases they carry.

While TBRF can infect dogs and deer as well as humans and is transmitted by tick bites, LBRF is transmitted from human to human via the body louse Pediculus humanus humanus. Very importantly, it is transmitted only by crushed infectious lice (Raoult et al [14], Badiaga et al [1]). We assume that only humans are hosts. A body louse can only become infected by Borrelia recurrentis bacteria and become a disease carrier if it feeds on the blood of an infectious human. The chemical reactions in the louse start with the ingestion of the bacteria within its midgut, where the bacteria multiply over a period of about six days. After that time, transmission to another human is possible if the infectious louse has been crushed dead. Crushing an infectious louse releases its infected body fluids onto the person's skin. The Borrelia recurrentis bacteria can penetrate intact mucosa and skin and enter the bloodstream, where they multiply (Southern and Sanford [16]). The transfer of bacteria to human skin can be via clothes or bedding and thus disease transmission from human to human is a possibility in situations where people share unwashed clothes or bedding that is soiled with the body fluids of crushed lice (Raoult et al [14], Badiaga et al [1]). An infectious living louse does not transmit LBRF disease when it bites a susceptible human, unless the louse is crushed. This is, however, a likely possibility because of the tendency of people to scratch their bites.

There have been numerous studies of vector-borne diseases associated with flying insects such as mosquitoes and midges. For example, the midge-borne disease bluetongue has been modelled and analysed by Gubbins et al [5], Hartemink et al [7], Gourley et al [4] and others. However, much less attention seems to have been given to the mathematical modelling of the dynamics of diseases transmitted by non-flying insects including LouseBorne Relapsing Fever. Whether the vectors can fly or not has some implications for the details of the modelling but many of the basic principles, including the way we model the latency stage, apply to both flying and non-flying vectors. There have been a few previous modelling studies specifically of lice populations. These include studies of head lice by Laguna and Risau-Gusman [11] who used Leslie and Lefkovitch matrices, body lice in sheep flocks (Horton and Carew [9]) and the recent PhD dissertation of Palmer [13] which is specifically on relapsing diseases. The most important difference between the model of LBRF in this paper, and recent models of diseases such as bluetongue that are carried by flying insects, is that the transmission of LBRF from a louse to a human 
involves the death of the louse. A living infectious louse does not transmit LBRF disease. The total biomass of dead lice is highly relevant, and we have a variable for it, since the infected body fluids from crushed lice are present on clothing and bedding.

This paper presents a detailed mathematical model, in the form of a system of seven delay differential equations, for the vector (lice) and host (human) populations. Delays enter into the model because of the need to take account of the latency times of the disease in both humans and lice. We formulate the model equations in Section 2 and then establish basic properties of the model. Further analysis including linear stability, dynamics of the disease and its persistence, and global stability are presented in Section 3. In Section 4, we discuss our findings.

\section{The model}

To formulate a mathematical model for louse-borne relapsing fever (LBRF), we introduce susceptible $S(t)$, exposed $E(t)$ and infectious $I(t)$ components for both hosts (humans) and vectors (lice). We apply subscripts $h$ and $v$ to denote hosts and vectors, respectively. We use an additional variable $C_{v}(t)$ to denote the biomass of crushed lice, because it is via crushed lice that human to human transmission occurs. We model the infection rate using mass action normalized by total host density $N_{h}(t)=S_{h}(t)+E_{h}(t)+I_{h}(t)$, with each transmission coefficient denoted by $\beta$ with appropriate subscripts $\left(\beta_{v h}\right.$ and $\beta_{h v}$ are the transmission coefficients for vector to human, and human to vector, respectively). The $E$ (exposed) variables take into account the latency stages of LBRF in humans and lice, which are modelled using distributed delays. A distributed delay formulation is appropriate for situations, such as this, in which the latency stage is not of a fixed duration. Its duration varies considerably between individuals and is usually between four and eight days in humans.

Let $\mathcal{F}_{v}(\eta)$ be the probability that a louse is still in the exposed class $\eta$ time units after becoming infected, having had a blood meal from an infectious human. Then $\mathcal{F}_{v}: \mathbb{R}_{+} \rightarrow \mathbb{R}_{+}$,

$$
\mathcal{F}_{v}(0)=1 \text { and } \mathcal{F}_{v}(\infty)=0 \text {. }
$$

We write $\mathcal{F}_{v}(\eta)$ in terms of a probability density function $f_{v}: \mathbb{R}_{+} \rightarrow \mathbb{R}_{+}$, where $f_{v}(r) \geq 0$ and

$$
\mathcal{F}_{v}(\eta)=\int_{\eta}^{\infty} f_{v}(r) d r .
$$

Note that

$$
\int_{0}^{\infty} f_{v}(r) d r=1 \quad \text { and } \quad \mathcal{F}_{v}^{\prime}(\eta)=-f_{v}(\eta)
$$

The probability of a louse remaining alive between times $\eta$ and $t$ is $\exp \left(-\left(\mu_{v}+c_{v}\right)(t-\eta)\right)$ where $\mu_{v}$ and $c_{v}$ are the per-capita rates at which lice die naturally or are crushed. Therefore, the total number of exposed lice at time $t$ is

$$
E_{v}(t)=\int_{-\infty}^{t} \beta_{h v} \frac{S_{v}(\xi) I_{h}(\xi)}{N_{h}(\xi)} e^{-\left(\mu_{v}+c_{v}\right)(t-\xi)} \mathcal{F}_{v}(t-\xi) d \xi
$$


where $\beta_{h v}$ is the host-vector infection rate, taken here as constant (the effects of seasonality will be considered in a separate work). In (2.1), the integral sums over all possible times $\xi \in(-\infty, t]$ of infection and the integrand is the number of new infections over an infinitesimal time interval $[\xi, \xi+d \xi]$, multiplied by the probability that those newly infected lice are still alive at time $t$ and by the probability $\mathcal{F}_{v}(t-\xi)$ that they are still in the exposed class.

With a similar formulation for the incubation period in humans, the total number of exposed humans is

$$
E_{h}(t)=\int_{-\infty}^{t} \beta_{v h} \frac{S_{h}(\xi) C_{v}(\xi)}{N_{h}(\xi)} e^{-\mu_{h}(t-\xi)} \mathcal{F}_{h}(t-\xi) d \xi
$$

where $\beta_{v h}$ is the vector-host transmission coefficient, $\mu_{h}$ is the per-capita mortality rate (due to causes other than LBRF) for humans and

$$
\mathcal{F}_{h}(\eta)=\int_{\eta}^{\infty} f_{h}(r) d r, \quad \text { with } \quad \int_{0}^{\infty} f_{h}(r) d r=1 \quad \text { and } \quad f_{h}(r) \geq 0 .
$$

Note that (2.2) makes the assumption that susceptible humans catch LBRF only from crushed infectious lice, so that the assumption of mass action leads to the product $S_{h}(\xi) C_{v}(\xi)$ in the integrand.

We propose the following equations for the human (subscript $h$ ) population:

$$
\begin{aligned}
& S_{h}^{\prime}(t)=b_{h}\left(\left(S_{h}+E_{h}+I_{h}\right)(t)\right)-\beta_{v h} \frac{S_{h}(t) C_{v}(t)}{N_{h}(t)}-\mu_{h} S_{h}(t)+\nu I_{h}(t) \\
& E_{h}^{\prime}(t)=\beta_{v h} \frac{S_{h}(t) C_{v}(t)}{N_{h}(t)}-\mu_{h} E_{h}(t)-\int_{-\infty}^{t} \beta_{v h} \frac{S_{h}(\xi) C_{v}(\xi)}{N_{h}(\xi)} e^{-\mu_{h}(t-\xi)} f_{h}(t-\xi) d \xi \\
& I_{h}^{\prime}(t)=\int_{-\infty}^{t} \beta_{v h} \frac{S_{h}(\xi) C_{v}(\xi)}{N_{h}(\xi)} e^{-\mu_{h}(t-\xi)} f_{h}(t-\xi) d \xi-\left(\mu_{h}+\delta_{h}+\nu\right) I_{h}(t),
\end{aligned}
$$

where $b_{h}\left(S_{h}(t)+E_{h}(t)+I_{h}(t)\right)$ is the birth rate for humans, $\nu$ is the per-capita recovery rate from LBRF, and $\delta_{h}$ is the human per-capita death rate due to LBRF.

Next we turn our attention to the lice population. We model this using a stagestructured approach, but there are differences between our approach here and most stagestructured models of populations that lump all pre-adult stages together and consider those as one compartment (often known as the larval stage, even though it includes all preadult stages), with the adults as the sole other compartment. It is an approach that works well for species in which larvae and adults have completely different characteristics and inhabit different environments, as is the case with mosquitoes. With lice, the situation is different. Larval and adult lice have remarkably similar characteristics and can be treated as indistinguishable as far as LBRF transmission is concerned. However, eggs do not transmit LBRF. Therefore, in our model it is the egg stage that is treated separately. All other life stages, including larvae and adults, are lumped together.

Lice do not transmit LBRF disease to their eggs. Newborns are always susceptible, and remain so throughout the egg stage. No variable for the number of eggs is necessary in the model. However, as soon as eggs hatch the lice larvae are at risk of infection 
immediately since they must feed on the host human blood. The time required for eggs to hatch is predictable and is therefore taken as a fixed constant $\tau$ for each egg. We let $\mu_{e}$ be the per-capita mortality rate for eggs. The probability of a louse surviving through the egg stage is therefore $\exp \left(-\mu_{e} \tau\right)$. Moreover, since LBRF does not cause death in lice, mortality of lice post hatching is caused either by crushing (at a per-capita rate $c_{v}$ ) or happens at a per-capita rate $\mu_{v}$ that accounts for all other causes of louse death. Therefore, we propose the following equations for the louse (subscript $v$, standing for vector) population:

$$
\begin{aligned}
S_{v}^{\prime}(t) & =e^{-\mu_{e} \tau} b_{v}\left(\left(S_{v}+E_{v}+I_{v}\right)(t-\tau)\right)-\beta_{h v} \frac{S_{v}(t) I_{h}(t)}{N_{h}(t)}-\left(\mu_{v}+c_{v}\right) S_{v}(t) \\
E_{v}^{\prime}(t) & =\beta_{h v} \frac{S_{v}(t) I_{h}(t)}{N_{h}(t)}-\left(\mu_{v}+c_{v}\right) E_{v}(t)-\int_{-\infty}^{t} \beta_{h v} \frac{S_{v}(\xi) I_{h}(\xi)}{N_{h}(\xi)} e^{-\left(\mu_{v}+c_{v}\right)(t-\xi)} f_{v}(t-\xi) d \xi \\
I_{v}^{\prime}(t) & =\int_{-\infty}^{t} \beta_{h v} \frac{S_{v}(\xi) I_{h}(\xi)}{N_{h}(\xi)} e^{-\left(\mu_{v}+c_{v}\right)(t-\xi)} f_{v}(t-\xi) d \xi-\left(\mu_{v}+c_{v}\right) I_{v}(t) \\
C_{v}^{\prime}(t) & =c_{v} I_{v}(t)-\mu_{b} C_{v}(t)
\end{aligned}
$$

where $b_{v}(\cdot)$ is the egg laying rate for lice, taken to be a function of the total number of lice and $\mu_{b}$ is the bacteria clearance rate from the crushed lice. All parameters are strictly positive except for $\tau$ which is nonnegative.

\section{Model analysis}

\subsection{Existence and uniqueness}

To determine the initial data that has to be prescribed, and to determine a suitable state space, one can consider what information would be required for the evaluation of the right hand sides of (2.3)-(2.9) at time $t=0$. Where delays are involved, initial data usually has to be prescribed on an interval, which may be $(-\infty, 0]$, and the interval in negative time over which data must be prescribed can differ from one component to the next, as is the case here. Moreover, the integral equations (2.1) and (2.2) impose constraints on the admissible initial data.

As the right hand sides of (2.3)-(2.9) clearly show, for the variables $S_{h}, S_{v}, E_{h}, I_{h}$ and $C_{v}$, initial data, denoted with superscript 0 , must be prescribed for all $t \leq 0$. We prescribe

$$
\begin{aligned}
& S_{h}(\theta)=S_{h}^{0}(\theta) \geq 0, \quad S_{v}(\theta)=S_{v}^{0}(\theta) \geq 0, \quad E_{h}(\theta)=E_{h}^{0}(\theta) \geq 0, \\
& I_{h}(\theta)=I_{h}^{0}(\theta) \geq 0, \quad C_{v}(\theta)=C_{v}^{0}(\theta) \geq 0, \quad \text { for } \theta \in(-\infty, 0] .
\end{aligned}
$$

The initial data for the variables $E_{v}$ and $I_{v}$ only needs to be given for $t \in[-\tau, 0]$ and thus we prescribe

$$
E_{v}(\theta)=E_{v}^{0}(\theta) \geq 0, \quad I_{v}(\theta)=I_{v}^{0}(\theta) \geq 0, \quad \theta \in[-\tau, 0] .
$$


The initial data for the seven variables must not only be prescribed as above but must additionally satisfy the constraints

$$
E_{v}^{0}(0)=\int_{-\infty}^{0} \beta_{h v} \frac{S_{v}^{0}(\xi) I_{h}^{0}(\xi)}{S_{h}^{0}(\xi)+E_{h}^{0}(\xi)+I_{h}^{0}(\xi)} e^{\left(\mu_{v}+c_{v}\right) \xi} \mathcal{F}_{v}(-\xi) d \xi
$$

and

$$
E_{h}^{0}(0)=\int_{-\infty}^{0} \beta_{v h} \frac{S_{h}^{0}(\xi) C_{v}^{0}(\xi)}{S_{h}^{0}(\xi)+E_{h}^{0}(\xi)+I_{h}^{0}(\xi)} e^{\mu_{h} \xi} \mathcal{F}_{h}(-\xi) d \xi
$$

which come from (2.1) and (2.2), evaluated at $t=0$. Note that, while constraint (3.12) is simply an expression for $E_{v}^{0}(0)$ in terms of the initial data for certain other variables, the second constraint (3.13) is an integral equation since the initial data for the variable $E_{h}$ is involved in both sides of the equation.

Let $B U C(-\infty, 0]$ be the set of bounded uniformly continuous functions on $(-\infty, 0]$. For a $\Delta>0$ to be chosen, define

$$
\mathcal{C}_{\Delta}=\left\{\phi:(-\infty, 0] \rightarrow \mathbb{R}: \phi(s) e^{\Delta s} \in B U C(-\infty, 0]\right\}
$$

with the norm

$$
\|\phi\|_{\mathcal{C}_{\Delta}}=\sup _{s \leq 0}\left|\phi(s) e^{\Delta s}\right|
$$

Then $\mathcal{C}_{\Delta}$ is a Banach space. For any particular $\xi \in(-\infty, 0]$,

$$
S_{h}^{0}(\xi) e^{\Delta \xi} \leq \sup _{\xi \leq 0}\left|S_{h}^{0}(\xi)\right| e^{\Delta \xi}=\left\|S_{h}^{0}\right\|_{\mathcal{C}_{\Delta}}
$$

and so

$$
S_{h}^{0}(\xi) \leq\left\|S_{h}^{0}\right\|_{\mathcal{C}_{\Delta}} e^{-\Delta \xi}
$$

and similarly for the other variables. To determine a suitable $\Delta$ for a viable state space, the integrals in the right hand sides of (2.5) and (2.8) must be finite for all $t \geq 0$ and, in particular, at $t=0$. So we consider the situation at $t=0$. The first of these integrals at $t=0$ is

$$
\begin{aligned}
\int_{-\infty}^{0} \beta_{v h} \frac{S_{h}^{0}(\xi) C_{v}^{0}(\xi)}{N_{h}^{0}(\xi)} e^{\mu_{h} \xi} f_{h}(-\xi) d \xi & \leq \int_{-\infty}^{0} \beta_{v h} C_{v}^{0}(\xi) e^{\mu_{h} \xi} f_{h}(-\xi) d \xi \\
& \leq\left\|C_{v}^{0}\right\|_{\mathcal{C}_{\Delta}} \int_{-\infty}^{0} \beta_{v h} e^{\left(\mu_{h}-\Delta\right) \xi} f_{h}(-\xi) d \xi
\end{aligned}
$$

using that $S_{h} / N_{h} \leq 1$. This expression is finite if $C_{v}^{0} \in \mathcal{C}_{\Delta}$ and $\Delta<\mu_{h}$, where we use that

$$
\int_{-\infty}^{0} f_{h}(-\xi) d \xi=\int_{0}^{\infty} f_{h}(\xi) d \xi=1
$$

If we estimate in the same way the integral arising in (2.8), and use that $I_{h} / N_{h} \leq 1$, we conclude finiteness of that integral at time $t=0$ if $S_{v}^{0} \in \mathcal{C}_{\Delta}$ and $\Delta<\mu_{v}+c_{v}$. Therefore to ensure finiteness of both integrals we choose $\Delta$ such that $0<\Delta<\min \left(\mu_{h}, \mu_{v}+c_{v}\right)$. With such a choice for $\Delta$ we can now construct a suitable state space in which to prove 
local existence of a unique solution. If additionally we can show that solutions remain bounded while they exist (sufficient conditions for this are presented later), then in fact we have global existence.

We use the subscript $t$ notation with its usual meaning in the theory of delay equations, i.e. $x_{t}$ is the function with values $x_{t}(\theta)=x(t+\theta), \theta \leq 0$. The precise domain for $\theta$ is either $(-\infty, 0]$ or $[-\tau, 0]$ depending on the solution component. The state of the system at time $t$ is that entity which contains all the information necessary to predict the solution at future times. For system (2.3)-(2.9), the state at time $t$ is

$$
S_{t}=\left(S_{h_{t}}, E_{h_{t}}, I_{h_{t}}, S_{v_{t}}, E_{v_{t}}, I_{v_{t}}, C_{v_{t}}\right)
$$

with the domains for the components being $(-\infty, 0]$ for all components except $E_{v}$ and $I_{v}$, for which it is $[-\tau, 0]$. In view of the various considerations above, the best choice for the state space, which we call $\mathcal{X}_{\Delta}$, is given as follows:

$$
S_{t}=\left(S_{h_{t}}, E_{h_{t}}, I_{h_{t}}, S_{v_{t}}, E_{v_{t}}, I_{v_{t}}, C_{v_{t}}\right) \in \mathcal{X}_{\Delta}=\mathcal{C}_{\Delta} \times \mathcal{C}_{\Delta} \times \mathcal{C}_{\Delta} \times \mathcal{C}_{\Delta} \times \mathcal{C}_{\tau} \times \mathcal{C}_{\tau} \times \mathcal{C}_{\Delta}
$$

where $\mathcal{C}_{\tau}$, which appears twice and relates to the components $E_{v}$ and $I_{v}$, is the Banach space of continuous functions from $[-\tau, 0]$ to $\mathbb{R}$ with the supremum norm denoted $\|\cdot\|_{\mathcal{C}_{\tau}}$.

The space $\mathcal{X}_{\Delta}$ is a Banach space with the norm

$$
\|S\|_{\mathcal{X}_{\Delta}}:=\max \left(\left\|S_{h}\right\|_{\mathcal{C}_{\Delta}},\left\|E_{h}\right\|_{\mathcal{C}_{\Delta}},\left\|I_{h}\right\|_{\mathcal{C}_{\Delta}},\left\|S_{v}\right\|_{\mathcal{C}_{\Delta}},\left\|E_{v}\right\|_{\mathcal{C}_{\tau}},\left\|I_{v}\right\|_{\mathcal{C}_{\tau}},\left\|C_{v}\right\|_{\mathcal{C}_{\Delta}}\right) .
$$

We will later establish that solution variables always remain non-negative, but for the purposes of establishing local existence of solutions it can be useful to have the birth functions defined even for negative arguments. Assume $b_{h}(\cdot)$ and $b_{v}(\cdot)$ are locally Lipschitz continuous for non-negative arguments. We extend the definitions of $b_{h}$ and $b_{v}$ to negative arguments by defining $b_{h}(N)=0=b_{v}(N)$ for $N<0$. Since the birth functions always satisfy $b_{h}(0)=b_{v}(0)=0$, such an extension preserves Lipschitz continuity. We are now in a position to prove the following theorem on local existence and uniqueness.

Theorem 1. Suppose the prescribed initial data $S_{0}=\left(S_{h_{0}}, E_{h_{0}}, I_{h_{0}}, S_{v_{0}}, E_{v_{0}}, I_{v_{0}}, C_{v_{0}}\right)$ meets the form and constraints given in (3.10)-(3.13) and that $S_{0} \in \mathcal{X}_{\Delta}$, with $0<\Delta<$ $\min \left(\mu_{h}, \mu_{v}+c_{v}\right)$. Suppose also that $b_{h}, b_{v}: \mathbb{R} \rightarrow \mathbb{R}$ are locally Lipschitz non-negative functions such that $b_{h}(0)=b_{v}(0)=0$. Then system (2.3)-(2.9) has a unique solution in $\mathcal{X}_{\Delta}$ defined on an interval $[0, T)$ for some $0<T \leq \infty$.

Proof. The proof essentially follows from well known standard theory, but we need to explain how we deal with the integral terms in system (2.3)-(2.9) since these all involve infinite delay. However, in each integral the contribution from the subinterval $\xi \in(-\infty, 0]$ only involves the initial data, which is given. Over any given finite interval of positive time, solutions of (2.3)-(2.9) can therefore be interpreted as solutions of another system with finite delay suitable for the application of well known results. To see how this works let us examine one particular equation with an integral term. Consider the $I_{v}$ equation (2.8). Let $t^{\#}$ be fixed but arbitrary. On the interval $t \in\left[0, t^{\#}\right]$, the variables satisfy a system of seven equations that includes the following equation with finite delay, replacing (2.8):

$$
I_{v}^{\prime}(t)=\varrho(t)+\int_{t-t^{\#}}^{t} \beta_{h v} \frac{S_{v}(\xi) I_{h}(\xi)}{N_{h}(\xi)} e^{-\left(\mu_{v}+c_{v}\right)(t-\xi)} f_{v}(t-\xi) d \xi-\left(\mu_{v}+c_{v}\right) I_{v}(t),
$$


where $\varrho(t):\left[0, t^{\#}\right] \rightarrow \mathbb{R}$ is the known function

$$
\varrho(t)=\int_{-\infty}^{t-t^{\#}} \beta_{h v} \frac{S_{v}^{0}(\xi) I_{h}^{0}(\xi)}{N_{h}^{0}(\xi)} e^{-\left(\mu_{v}+c_{v}\right)(t-\xi)} f_{v}(t-\xi) d \xi, \quad t \in\left[0, t^{\#}\right] .
$$

The integral terms in the other equations of (2.3)-(2.9) are dealt with similarly. In this way, we convert the original system to one with finite delay. The function $\varrho(t)$ is well defined and continuous, since the initial data is in $\mathcal{X}_{\Delta}$. On subintervals of $\left[0, t^{\#}\right]$, existence and uniqueness of solutions follows from standard theory (Hale and Verduyn Lunel [6]).

\subsection{Positivity and boundedness}

Non-negativity of solutions can be established easily using an approach that is now standard. The rigor of this approach has been well established (see, for example, Theorem 5.2.1 on page 81 of Smith [15]). The approach is applied to system (2.3)-(2.9) in the following manner. In each equation, except for the $E_{h}$ and $E_{v}$ equations, we identify the variable that appears in its left hand side. Then, every undelayed occurrence of that variable in the right hand side is set equal to zero. If what remains of the right hand side is non-negative when all remaining variables (delayed and undelayed) are non-negative, then non-negativity follows. For example, in the $S_{h}$ equation (equation (2.3)), we set all $S_{h}(t)$ terms in the right hand side to zero leaving

$$
S_{h}^{\prime}(t)=b_{h}\left(\left(E_{h}+I_{h}\right)(t)\right)+\nu I_{h}(t) \geq 0
$$

when $E_{h}(t), I_{h}(t) \geq 0$. This test will not establish non-negativity of the $E_{h}$ and $E_{v}$ variables. However, non-negativity of those variables follows from the fact that we have integral equations for them (see equations (2.1) and (2.2)).

The above approach only establishes non-negativity of solution components. Establishing that solution components become and remain strictly positive is more difficult and, as we shall see, it raises a number of delicate issues. We start by establishing strict positivity of $I_{h}(t)$ and $I_{v}(t)$ under certain assumptions. With the help of this, we then identify the class of initial data that results in all seven variables becoming and remaining strictly positive. It is the nature of our problem, and in particular its dependence on distributed delay terms with relatively general delay kernels $f_{h}(t)$ and $f_{v}(t)$, that makes this issue non-trivial.

Proposition 1. Assume that $f_{h}$ and $f_{v}$ are continuous and that, for some $\eta^{*} \leq 0$, either:

(i) $S_{h}\left(\eta^{*}\right) C_{v}\left(\eta^{*}\right)>0$ and $f_{h}\left(\left[-\eta^{*}, \infty\right)\right) \neq\{0\}$, or

(ii) $S_{v}\left(\eta^{*}\right) I_{h}\left(\eta^{*}\right)>0$ and $f_{v}\left(\left[-\eta^{*}, \infty\right)\right) \neq\{0\}$,

in either case with the initial functions being continuous. Then $I_{h}(t)$ or $I_{v}(t)$, respectively, becomes strictly positive at some time, and remains strictly positive as long as the solution exists. 
Proof. We prove only the first statement of the proposition. Since $f_{h}\left(\left[-\eta^{*}, \infty\right)\right) \neq\{0\}$, there exists $\xi^{*} \geq-\eta^{*}$ such that $f_{h}\left(\xi^{*}\right)>0$ and, since $f_{h}$ is a continuous function, $f_{h}(\xi)>0$ in some interval of $\xi^{*}$. Moreover, we can arrange so that $\xi^{*}>-\eta^{*}$. If $\xi$ is close enough to $\eta^{*}$, more precisely, if $\xi$ is in some open interval $I_{1}$ containing $\eta^{*}$, then $f_{h}\left(\xi^{*}+\eta^{*}-\xi\right)>0$.

Now suppose that $I_{h}(t) \equiv 0$ for all $t>0$. Then

$$
\int_{-\infty}^{t} \beta_{v h} \frac{S_{h}(\xi) C_{v}(\xi)}{N_{h}(\xi)} e^{-\mu_{h}(t-\xi)} f_{h}(t-\xi) d \xi \equiv 0 \text { for all } t>0 .
$$

The above integral evaluated at time $t=\xi^{*}+\eta^{*}>0$ is

$$
\int_{-\infty}^{\xi^{*}+\eta^{*}} \beta_{v h} \frac{S_{h}(\xi) C_{v}(\xi)}{N_{h}(\xi)} e^{-\mu_{h}\left(\xi^{*}+\eta^{*}-\xi\right)} f_{h}\left(\xi^{*}+\eta^{*}-\xi\right) d \xi .
$$

Since $S_{h}\left(\eta^{*}\right) C_{v}\left(\eta^{*}\right)>0$, and $S_{h}(t)$ and $C_{v}(t)$ are continuous, there exists some interval $I_{2}$ containing $\eta^{*}$ such that $S_{h}(\xi) C_{v}(\xi)>0$ for all $\xi \in I_{2}$. But,

$$
\begin{aligned}
& \int_{-\infty}^{\xi^{*}+\eta^{*}} \beta_{v h} \frac{S_{h}(\xi) C_{v}(\xi)}{N_{h}(\xi)} e^{-\mu_{h}\left(\xi^{*}+\eta^{*}-\xi\right)} f_{h}\left(\xi^{*}+\eta^{*}-\xi\right) d \xi \\
& \geq \int_{I_{1} \cap I_{2}} \beta_{v h} \frac{S_{h}(\xi) C_{v}(\xi)}{N_{h}(\xi)} e^{-\mu_{h}\left(\xi^{*}+\eta^{*}-\xi\right)} f_{h}\left(\xi^{*}+\eta^{*}-\xi\right) d \xi>0 .
\end{aligned}
$$

This contradicts $I_{h}(t) \equiv 0$. Hence, $I_{h}(t)$ must become strictly positive at some time $t>0$. It remains positive thereafter, since it satisfies the differential inequality $I_{h}^{\prime}(t) \geq$ $-\left(\mu_{h}+\delta_{h}+\nu\right) I_{h}(t)$.

The next question that arises is the following: is it true that if one of $I_{h}(t)$ or $I_{v}(t)$ becomes and remains positive, then all other variables necessarily become and remain positive? We see immediately that, mathematically, this need not follow. There is a scenario in which $I_{h}(t)>0$ but $I_{v}(t)$ remains identically zero for all $t \in \mathbb{R}$, but it is an exceedingly implausible scenario biologically. The scenario is that there are no living lice (susceptible, exposed or infectious) at all, but there are some crushed lice. It is easy to appreciate that the number of infectious humans $I_{h}(t)$ would become and remain positive. However, the numbers of susceptible, exposed and infectious lice could all remain identically zero for all time. Even in this exceedingly unlikely scenario, $C_{v}(t)$ will tend to zero as $t \rightarrow \infty$ and the system evolves to a steady state in which there are only susceptible humans, the number $S_{h}(t)$ of which satisfies

$$
S_{h}^{\prime}(t)=b_{h}\left(S_{h}(t)\right)-\mu_{h} S_{h}(t) .
$$

Note also that the model (2.3)-(2.9) does not make sense if there are no humans at all, since the infection rates are divided by total host (human) density $N_{h}(t)$. Therefore, we do not consider the scenario in which there are lice but no humans.

Our next proposition aims to capture the minimal assumptions on the initial data which will ensure that all seven variables become and remain strictly positive. Assumption (ii) implies there are some humans present initially. The variables need not all become positive at the same time. 
Proposition 2. Suppose that $b_{h}(\cdot)$ and $b_{v}(\cdot)$ satisfy $b_{h}(0)=0$ and $b_{v}(0)=0$, and are otherwise strictly positive. Suppose also that $f_{h}$ and $f_{v}$ are continuous and that, for some $\eta^{*} \leq 0$,

(i) $S_{v}\left(\eta^{*}\right) I_{h}\left(\eta^{*}\right)>0$ and $f_{v}\left(\left[-\eta^{*}, \infty\right)\right) \neq\{0\}$,

(ii) at least one of $S_{h}(0), E_{h}(0), I_{h}(0)$ is strictly positive.

Then all seven variables of system (2.3)-(2.9) become and remain strictly positive.

Proof. By Proposition 1, $I_{v}(t)$ becomes strictly positive at some time $t \geq 0$, and remains strictly positive. Equation (2.9) clearly implies that $C_{v}(t)$ can not remain identically zero for all $t \geq 0$, therefore, $C_{v}(t)>0$ for all $t$ sufficiently large. Suppose now that $S_{v}(t) \equiv 0$ for all $t \geq 0$. Then (2.6) implies that

$$
0=e^{-\mu_{e} \tau} b_{v}\left(\left(S_{v}+E_{v}+I_{v}\right)(t-\tau)\right)
$$

so that $\left(S_{v}+E_{v}+I_{v}\right)(t-\tau) \equiv 0$ for all $t \geq 0$, which is only true only if $S_{v}, E_{v}, I_{v} \equiv 0$ for all $t \geq-\tau$, contradicting that $I_{v}$ becomes and remains strictly positive. Therefore, $S_{v}(t)$ must become positive at some time $t_{1}$. For $t>t_{1}$,

$$
S_{v}^{\prime}(t) \geq-S_{v}(t)\left(\beta_{h v} \frac{I_{h}(t)}{N_{h}(t)}+\mu_{v}+c_{v}\right)
$$

so that

$$
S_{v}(t) \geq S_{v}\left(t_{1}\right) \exp \left\{-\int_{t_{1}}^{t}\left(\beta_{h v} \frac{I_{h}(\xi)}{N_{h}(\xi)}+\mu_{v}+c_{v}\right) d \xi\right\}>0 .
$$

Next we establish strict positivity of $E_{v}(t)$. Recall that

$$
E_{v}(t)=\int_{-\infty}^{t} \beta_{h v} \frac{S_{v}(\xi) I_{h}(\xi)}{N_{h}(\xi)} e^{-\left(\mu_{v}+c_{v}\right)(t-\xi)} \int_{t-\xi}^{\infty} f_{v}(r) d r d \xi
$$

Since $f_{v}\left(\left[-\eta^{*}, \infty\right)\right) \neq\{0\}$, there exists $\xi_{v}^{*} \geq-\eta^{*}$ such that $f_{v}\left(\xi_{v}^{*}\right)>0$ and we may choose $\xi_{v}^{*}$ such that $\xi_{v}^{*}>-\eta^{*}$. If $\xi$ is in some open interval $I_{1}$ containing $\eta^{*}, f_{v}\left(\xi_{v}^{*}+\eta^{*}-\xi\right)>0$. Also, since $S_{v}$ and $I_{h}$ are continuous there is an interval $I_{2}$ containing $\eta^{*}$ such that

$$
S_{v}(\xi) I_{h}(\xi)>0 \quad \text { for all } \xi \in I_{2} \text {. }
$$

Now evaluate $E_{v}(t)$ at time $t=\xi_{v}^{*}+\eta^{*}$ :

$$
\begin{aligned}
E_{v}\left(\xi_{v}^{*}+\eta^{*}\right) & =\int_{-\infty}^{\xi_{v}^{*}+\eta^{*}} \beta_{h v} \frac{S_{v}(\xi) I_{h}(\xi)}{N_{h}(\xi)} e^{-\left(\mu_{v}+c_{v}\right)\left(\xi_{v}^{*}+\eta^{*}-\xi\right)} \int_{\xi_{v}^{*}+\eta^{*}-\xi}^{\infty} f_{v}(r) d r d \xi \\
& \geq \int_{I_{1} \cap I_{2}} \beta_{h v} \frac{S_{v}(\xi) I_{h}(\xi)}{N_{h}(\xi)} e^{-\left(\mu_{v}+c_{v}\right)\left(\xi_{v}^{*}+\eta^{*}-\xi\right)} \int_{\xi_{v}^{*}+\eta^{*}-\xi}^{\infty} f_{v}(r) d r d \xi
\end{aligned}
$$

Note that $S_{v}(\xi) I_{h}(\xi)>0$ on $I_{1} \cap I_{2}$. Also, $\xi \in I_{1} \cap I_{2} \subset I_{1}$ so $f_{v}\left(\xi_{v}^{*}+\eta^{*}-\xi\right)>0$, i.e., $f_{v}(r)$ is strictly positive at the lower limit of integration $r=\xi_{v}^{*}+\eta^{*}-\xi$ on the inner 
integral. Also, $f_{v}(r)$ is continuous and non-negative everywhere. Taken together, these facts imply that

$$
\int_{\xi_{v}^{*}+\eta^{*}-\xi}^{\infty} f_{v}(r) d r>0
$$

and therefore $E_{v}\left(\xi_{v}^{*}+\eta^{*}\right)>0$ and $E_{v}$ will remain strictly positive after time $\xi_{v}^{*}+\eta^{*}$.

Next we prove that $S_{h}, E_{h}, I_{h}$ become and remain positive. We know by hypothesis that at least one of $S_{h}(0), E_{h}(0), I_{h}(0)>0$. Suppose $S_{h}(t) \equiv 0$ for all $t>0$. Then $(2.3)$ implies

$$
0=b_{h}\left(\left(S_{h}+E_{h}+I_{h}\right)(t)\right)+\nu I_{h}(t),
$$

which yields that $I_{h}(t) \equiv 0$ and $b_{h}\left(\left(S_{h}+E_{h}+I_{h}\right)(t)\right) \equiv 0$, so that $\left(S_{h}+E_{h}+I_{h}\right)(t) \equiv 0$ and, in particular, $S_{h}(t) \equiv 0$, a contradiction.

Proving that $E_{h}(t)$ becomes and remains positive is similar to the corresponding proof for $E_{v}(t)$ shown above.

The proof that $I_{h}(t)$ becomes and remains positive is similar to the corresponding proof for $I_{v}(t)$ in Proposition 1, but here we do not need to worry about the condition $S_{h}\left(\eta^{*}\right) C_{v}\left(\eta^{*}\right)>0$. That condition holds (with some translate in time) since we have strict positivity of $S_{h}$ and $C_{v}$ for $t$ sufficiently large. Therefore, $I_{h}(t)$ will become and remain positive under the condition

$$
\int_{0}^{\infty} f_{h}(t) d t=1
$$

because that condition (together with continuity of $f_{h}$ ) implies the existence of an interval of values in which $f_{h}(t)>0$, as in the proof of Proposition 1.

To ensure that solutions are bounded we first establish the following lemma. It is a rather general result because it does not require $b$ to be either monotone or bounded. It admits birth functions that grow, as long as they meet the requirement that $b(S)<\mu S$ for sufficiently large $S$.

Lemma 1. Let $b: \mathbb{R}_{+} \rightarrow \mathbb{R}_{+}$be a continuous function. Assume that $\mu>0$ is such that there are some values of $S$ for which $b(S)>\mu S$, but there exists $S^{0}>0$ such that

$$
b\left(S^{0}\right)=\mu S^{0} \quad \text { and } \quad b(S)<\mu S \quad \text { for all } S>S^{0} .
$$

Define

$$
\bar{b}(S)=\sup _{0 \leq s \leq S} b(s) .
$$

Then $\bar{b}(S)$ is monotone non-decreasing, continuous, and

$$
b(S) \leq \bar{b}(S) \forall S \geq 0 .
$$

Moreover, if $\breve{S}=b^{0} / \mu$, where $b^{0}=\bar{b}\left(S^{0}\right)$, then $\breve{S} \geq S^{0}$ and $\bar{b}(S)<\mu S$ for all $S>\breve{S}$. Moreover, if $b$ is monotone non-decreasing on $\left[0, S^{0}\right]$, then $\breve{S}=S^{0}$. 
Proof. We prove only that $\breve{S} \geq S^{0}$, and that $\bar{b}(S)<\mu S$ for all $S>\breve{S}$. The truth of the other statements of the lemma is clear.

Suppose, for contradiction, that $\breve{S}<S^{0}$. Then $\bar{b}\left(S^{0}\right)<\mu S^{0}=b\left(S^{0}\right)$. However, from the definition of $\bar{b}$, we should have $b\left(S^{0}\right) \leq \bar{b}\left(S^{0}\right)$.

Suppose, for contradiction, that there exists $S^{*}>\breve{S}$ such that $\bar{b}\left(S^{*}\right) \geq \mu S^{*}$. Then

$$
\begin{aligned}
\bar{b}\left(S^{*}\right) & =\sup _{0 \leq s \leq S^{*}} b(s)=\max \left\{\sup _{0 \leq s \leq S^{0}} b(s), \sup _{S^{0}<s \leq S^{*}} b(s)\right\} \\
& <\max \left\{\sup _{0 \leq s \leq S^{0}} b(s), \sup _{S^{0}<s \leq S^{*}} \mu s\right\}=\max \left\{\bar{b}\left(S^{0}\right), \mu S^{*}\right\} \\
& =\max \left\{\mu \breve{S}, \mu S^{*}\right\}=\mu S^{*},
\end{aligned}
$$

a contradiction.

We also require the following lemma, closely related to Proposition 3.3 in Gourley, Thieme and van den Driessche [4].

Lemma 2. Assume that the hypotheses of Lemma 1 hold, and let $\breve{S}=b^{0} / \mu$ where $b^{0}=\bar{b}\left(S^{0}\right)$. Let $N(t)$ be differentiable on $(0, \infty)$ and satisfy

$$
N^{\prime}(t) \leq b(N(t-\tau))-\mu N(t)
$$

Then

$$
\limsup _{t \rightarrow \infty} N(t) \leq \breve{S}
$$

Moreover, if $b$ is monotone non-decreasing on $\left[0, S^{0}\right]$, then $\breve{S}=S^{0}$.

With the above results we may prove the following theorem.

Theorem 2. Suppose that $b_{v}$ and $b_{h}$ are continuous functions. Assume that there exist $N_{v}^{0}>0$ and $N_{h}^{0}>0$ such that

$$
\begin{gathered}
e^{-\mu_{e} \tau} b_{v}\left(N_{v}^{0}\right)=\left(\mu_{v}+c_{v}\right) N_{v}^{0} \quad \text { and } \quad e^{-\mu_{e} \tau} b_{v}(N)<\left(\mu_{v}+c_{v}\right) N \quad \text { for all } N>N_{v}^{0} ; \\
b_{h}\left(N_{h}^{0}\right)=\mu_{h} N_{h}^{0} \quad \text { and } \quad b_{h}(N)<\mu_{h} N \quad \text { for all } N>N_{h}^{0} .
\end{gathered}
$$

Define

$$
\bar{b}_{v}(N)=\sup _{0 \leq \eta \leq N} b_{v}(\eta) \text { and } \bar{b}_{h}(N)=\sup _{0 \leq \eta \leq N} b_{h}(\eta)
$$

and

$$
\begin{gathered}
\breve{N}_{v}=b_{v}^{0} /\left(\mu_{v}+c_{v}\right), \text { where } b_{v}^{0}=\bar{b}_{v}\left(N_{v}^{0}\right) \\
\breve{N}_{h}=b_{h}^{0} / \mu_{h}, \text { where } b_{h}^{0}=\bar{b}_{h}\left(N_{h}^{0}\right)
\end{gathered}
$$

Then

$$
\limsup _{t \rightarrow \infty}\left(S_{v}(t)+E_{v}(t)+I_{v}(t)\right) \leq \breve{N}_{v}
$$

and

$$
\limsup _{t \rightarrow \infty}\left(S_{h}(t)+E_{h}(t)+I_{h}(t)\right) \leq \breve{N}_{h}
$$

Moreover, if $b_{v}$ is monotone non-decreasing on $\left[0, N_{v}^{0}\right]$ then $\breve{N}_{v}=N_{v}^{0}$, and if $b_{h}$ is monotone non-decreasing on $\left[0, N_{h}^{0}\right]$ then $\breve{N}_{h}=N_{h}^{0}$. 
Proof. The total number of lice $N_{v}(t)=S_{v}(t)+E_{v}(t)+I_{v}(t)$ satisfies

$$
\frac{d N_{v}(t)}{d t}=e^{-\mu_{e} \tau} b_{v}\left(N_{v}(t-\tau)\right)-\left(\mu_{v}+c_{v}\right) N_{v}(t),
$$

and therefore (3.15) follows from an application of Lemmas 1 and 2. The total number of humans $N_{h}(t)=S_{h}(t)+E_{h}(t)+I_{h}(t)$ satisfies

$$
\frac{d N_{h}(t)}{d t}=b_{h}\left(N_{h}(t)\right)-\mu_{h} N_{h}(t)-\delta_{h} I_{h}(t) \leq b_{h}\left(N_{h}(t)\right)-\mu_{h} N_{h}(t),
$$

and (3.16) follows from another application of those two lemmas.

\subsection{Existence of disease-free equilibria}

System (2.3)-(2.9) may have a disease-free equilibrium in which the $E$ and $I$ variables, and $C_{v}$, are all zero while $S_{h}=S_{h}^{0}, S_{v}=S_{v}^{0}$, where $S_{h}^{0}$ and $S_{v}^{0}$ satisfy

$$
\begin{aligned}
b_{h}\left(S_{h}^{0}\right) & =\mu_{h} S_{h}^{0}, \\
e^{-\mu_{e} \tau} b_{v}\left(S_{v}^{0}\right) & =\left(\mu_{v}+c_{v}\right) S_{v}^{0} .
\end{aligned}
$$

The existence of unique values $S_{h}^{0}>0$ and $S_{v}^{0}>0$ satisfying these equations depends on the birth rate functions $b_{h}(\cdot)$ and $b_{v}(\cdot)$ and the values of the model parameters that appear in (3.17)-(3.18). We are assured of the existence of unique $S_{h}^{0}>0$ and $S_{v}^{0}>0$ under assumptions (A1) and (A2) below. These assumptions essentially state that the birth (or maturation) rate exceeds the death rate at lower densities, but that deaths outweigh births at high densities since competition effects then become important and tend to reduce fecundity. These are minimal, yet reasonable, assumptions for any population in a habitat where conditions are right for it to thrive, yet there are factors that limit further growth at high densities.

(A1) $b_{h}\left(S_{h}\right)$ is nonnegative with $b_{h}(0)=0$ and there exists $S_{h}^{0}>0$ such that $b_{h}\left(S_{h}\right)>\mu_{h} S_{h}$ when $0<S_{h}<S_{h}^{0}$ and $b_{h}\left(S_{h}\right)<\mu_{h} S_{h}$ when $S_{h}^{0}<S_{h}$.

(A2) $b_{v}\left(S_{v}\right)$ is nonnegative with $b_{v}(0)=0$ and there exists $S_{v}^{0}>0$ such that $e^{-\mu_{e} \tau} b_{v}\left(S_{v}\right)>\left(\mu_{v}+c_{v}\right) S_{v}$ when $0<S_{v}<S_{v}^{0}$ and $e^{-\mu_{e} \tau} b_{v}\left(S_{v}\right)<\left(\mu_{v}+c_{v}\right) S_{v}$ when $S_{v}^{0}<S_{v}$.

Assumptions (A1) and (A2) are not required for all our results but we shall impose them as necessary.

\subsection{Extinction of the whole lice population}

From (3.18) note that, as the per-capita crushing rate $c_{v}$ increases, the steady state component $S_{v}^{0}$ decreases. For realistic $b_{v}(\cdot)$, if $c_{v}$ is sufficiently large equation (3.18) has no root with $S_{v}^{0}>0$. This suggests the following result on extinction (in infinite time) of the whole lice population. 
Theorem 3. If $b_{v}(0)=0$ and $b_{v}(\cdot)$ is non-negative, concave, twice differentiable and such that

$$
\mu_{v}+c_{v}>e^{-\mu_{e} \tau} b_{v}^{\prime}(0)
$$

then, for sufficiently large $c_{v}$, the whole lice population $N_{v}(t) \rightarrow 0$ as $t \rightarrow \infty$.

Proof. Let $N_{v}(t)=S_{v}(t)+E_{v}(t)+I_{v}(t)$ be the total number of lice. Then

$$
\frac{d N_{v}(t)}{d t}=e^{-\mu_{e} \tau} b_{v}\left(N_{v}(t-\tau)\right)-\left(\mu_{v}+c_{v}\right) N_{v}(t) .
$$

Since $b_{v}(\cdot)$ is concave, a Taylor expansion gives $b_{v}\left(N_{v}\right) \leq b_{v}^{\prime}(0) N_{v}$ and therefore, from (3.19),

$$
\frac{d N_{v}(t)}{d t} \leq e^{-\mu_{e} \tau} b_{v}^{\prime}(0) N_{v}(t-\tau)-\left(\mu_{v}+c_{v}\right) N_{v}(t) .
$$

The right hand side of (3.20) increases as $N_{v}(t-\tau)$ increases, since $b_{v}^{\prime}(0)>0$, and this facilitates the application of a comparison argument. Let $\tilde{N}_{v}(t)$ be the solution of

$$
\frac{d \tilde{N}_{v}(t)}{d t}=e^{-\mu_{e} \tau} b_{v}^{\prime}(0) \tilde{N}_{v}(t-\tau)-\left(\mu_{v}+c_{v}\right) \tilde{N}_{v}(t)
$$

subject to the same initial data as the variable $N_{v}(t)$. By Theorem 5.1.1 on page 78 of [15], $N_{v}(t) \leq \tilde{N}_{v}(t)$. Since $e^{-\mu_{e} \tau} b_{v}^{\prime}(0)<\mu_{v}+c_{v}, \tilde{N}_{v}(t) \rightarrow 0$ as $t \rightarrow \infty$. Since $0 \leq N_{v}(t) \leq \tilde{N}_{v}(t), N_{v}(t) \rightarrow 0$ as $t \rightarrow \infty$.

We now know that if the lice crushing rate $c_{v}$ is increased to a sufficiently large value, the whole lice population becomes extinct. Lowering the crushing rate will increase a positive $S_{v}^{0}$ but a sufficiently low crushing rate, as we show later, always eradicates LBRF. This is because LBRF transmission is only via crushed lice. In fact, LBRF can be eradicated either by taking $c_{v}$ sufficiently low or sufficiently large (in the latter case because the lice themselves become extinct).

\subsection{Linear stability of the disease-free equilibrium}

Assume (A1) and (A2) hold, so that there exists a disease-free equilibrium with $S_{h}^{0}>0$ and $S_{v}^{0}>0$ and all other components zero. We analyse the linear stability of this equilibrium by linearising the system about it. We introduce small perturbations (the tilde quantities) defined by

$$
\begin{gathered}
S_{h}=S_{h}^{0}+\tilde{S}_{h}, \quad E_{h}=0+\tilde{E}_{h}, \quad I_{h}=0+\tilde{I}_{h} \\
S_{v}=S_{v}^{0}+\tilde{S}_{v}, \quad E_{v}=0+\tilde{E}_{v}, \quad I_{v}=0+\tilde{I}_{v}, \quad C_{v}=0+\tilde{C}_{v} .
\end{gathered}
$$

It turns out that the linearisations of the $I_{h}, I_{v}$ and $C_{v}$ equations of (2.3)-(2.9) form a decoupled subsystem that can be used to show that those variables approach zero under some conditions. Therefore, we start by considering the $S_{h}$ and $S_{v}$ equations and their linearisations in the case when $I_{h}=I_{v}=C_{v}=E_{h}=E_{v}=0$. As a starting point we prove the following theorem, which admits only perturbations in which no disease is introduced. Later we prove Theorem 5 which admits general small perturbations (including small introductions of disease). 
Theorem 4. If (A1) and (A2) hold, $b_{h}^{\prime}\left(S_{h}^{0}\right)<\mu_{h}$ and

$$
-\left(\mu_{v}+c_{v}\right) \leq e^{-\mu_{e} \tau} b_{v}^{\prime}\left(S_{v}^{0}\right)<\mu_{v}+c_{v},
$$

then the disease free equilibrium in which $S_{h}=S_{h}^{0}$ and $S_{v}=S_{v}^{0}$ is locally asymptotically stable to small perturbations in which $C_{v}$ and all $E$ and I variables remain zero.

Proof. With $C_{v}$ and the $E$ and $I$ variables remaining zero, the $S_{h}$ equation linearises as follows:

$$
\tilde{S}_{h}^{\prime}(t)=\tilde{S}_{h}(t) b_{h}^{\prime}\left(S_{h}^{0}\right)-\mu_{h} \tilde{S}_{h}(t) .
$$

Thus, since $b_{h}^{\prime}\left(S_{h}^{0}\right)<\mu_{h}, \tilde{S}_{h}(t) \rightarrow 0$ and therefore $S_{h}(t) \rightarrow S_{h}^{0}$ as $t \rightarrow \infty$. Linearising the $S_{v}$ equation gives

$$
\tilde{S}_{v}^{\prime}(t)=e^{-\mu_{e} \tau} \tilde{S}_{v}(t-\tau) b_{v}^{\prime}\left(S_{v}^{0}\right)-\left(\mu_{v}+c_{v}\right) \tilde{S}_{v}(t)
$$

and the ansatz $\tilde{S}_{v}(t)=e^{\lambda t}$ leads to the characteristic equation

$$
\lambda+\mu_{v}+c_{v}=e^{-\mu_{e} \tau} b_{v}^{\prime}\left(S_{v}^{0}\right) e^{-\lambda \tau} .
$$

Assumption (3.21) implies that the roots of (3.23) all have negative real parts, as we now show. Suppose, for a contradiction, that there exists a root $\lambda^{*}$ of (3.23) such that $\operatorname{Re} \lambda^{*} \geq 0$. From (3.23),

$$
\left|\lambda^{*}+\mu_{v}+c_{v}\right|=e^{-\mu_{e} \tau}\left|b_{v}^{\prime}\left(S_{v}^{0}\right)\right| e^{-\left(\operatorname{Re} \lambda^{*}\right) \tau} \leq e^{-\mu_{e} \tau}\left|b_{v}^{\prime}\left(S_{v}^{0}\right)\right| .
$$

Hence $\lambda^{*}$ lies in the disk in $\mathbb{C}$ of radius $e^{-\mu_{e} \tau}\left|b_{v}^{\prime}\left(S_{v}^{0}\right)\right|$, centered at the point $-\left(\mu_{v}+c_{v}\right) \in \mathbb{C}$. But if

$$
-\left(\mu_{v}+c_{v}\right)<e^{-\mu_{e} \tau} b_{v}^{\prime}\left(S_{v}^{0}\right)<\mu_{v}+c_{v},
$$

then this disk is contained entirely in the open left side of complex plane so that $\operatorname{Re} \lambda^{*}<0$, contradicting $\operatorname{Re} \lambda^{*} \geq 0$. However, assumption (3.21) allows the possibility that

$$
-\left(\mu_{v}+c_{v}\right)=e^{-\mu_{e} \tau} b_{v}^{\prime}\left(S_{v}^{0}\right),
$$

in which case the above-mentioned disk is in $\{\operatorname{Re} \lambda<0\} \cup\{0\}$, requiring us to consider the possibility that $\lambda^{*}=0$. In that case, from (3.23),

$$
\mu_{v}+c_{v}=e^{-\mu_{e} \tau} b_{v}^{\prime}\left(S_{v}^{0}\right)=-\left(\mu_{v}+c_{v}\right),
$$

a contradiction. Hence, Re $\lambda<0$ for all roots of the characteristic equation (3.23).

Next, we note that the linearisations of the $I_{h}, I_{v}, C_{v}$ equations form a self contained subsystem determining those three variables (near to the disease-free equilibrium). More precisely, the small variables $\tilde{I}_{h}, \tilde{I}_{v}$ and $\tilde{C}_{v}$ are determined by

$$
\begin{aligned}
\tilde{I}_{h}^{\prime}(t) & =\int_{-\infty}^{t} \beta_{v h} \tilde{C}_{v}(\xi) e^{-\mu_{h}(t-\xi)} f_{h}(t-\xi) d \xi-\left(\mu_{h}+\delta_{h}+\nu\right) \tilde{I}_{h}(t), \\
\tilde{I}_{v}^{\prime}(t) & =\int_{-\infty}^{t} \beta_{h v}\left(\frac{S_{v}^{0}}{S_{h}^{0}}\right) \tilde{I}_{h}(\xi) e^{-\left(\mu_{v}+c_{v}\right)(t-\xi)} f_{v}(t-\xi) d \xi-\left(\mu_{v}+c_{v}\right) \tilde{I}_{v}(t), \\
\tilde{C}_{v}^{\prime} & (t)=c_{v} \tilde{I}_{v}(t)-\mu_{b} \tilde{C}_{v}(t) .
\end{aligned}
$$


Seeking non-trivial solutions of the form $\left(\tilde{I}_{h}(t), \tilde{I}_{v}(t), \tilde{C}_{v}(t)\right)=e^{\lambda t}\left(c_{1}, c_{2}, c_{3}\right)$, we find that $\lambda$ must satisfy a characteristic equation that is most easily studied when written in the form

$$
\lambda+\mu_{b}=\frac{\beta_{v h} \beta_{h v} c_{v} S_{v}^{0} \hat{f}_{h}\left(\lambda+\mu_{h}\right) \hat{f}_{v}\left(\lambda+\mu_{v}+c_{v}\right)}{S_{h}^{0}\left(\lambda+\mu_{h}+\delta_{h}+\nu\right)\left(\lambda+\mu_{v}+c_{v}\right)}
$$

where $\hat{f}_{h}$ and $\hat{f}_{v}$ are the Laplace transforms of $f_{h}$ and $f_{v}$ :

$$
\hat{f}_{h}(\lambda)=\int_{0}^{\infty} e^{-\lambda \omega} f_{h}(\omega) d \omega, \quad \hat{f}_{v}(\lambda)=\int_{0}^{\infty} e^{-\lambda \omega} f_{v}(\omega) d \omega .
$$

We investigate the roots of (3.24) in the next theorem. We denote by $R_{0}$ the basic reproduction number for the disease. It takes the form

$$
R_{0}=\frac{\beta_{v h} \beta_{h v} c_{v} S_{v}^{0} \hat{f}_{h}\left(\mu_{h}\right) \hat{f}_{v}\left(\mu_{v}+c_{v}\right)}{S_{h}^{0}\left(\mu_{h}+\delta_{h}+\nu\right)\left(\mu_{v}+c_{v}\right) \mu_{b}} .
$$

Later, we shall be introducing other basic reproduction numbers $R_{0}^{h}$ and $R_{0}^{v}$ which relate to the survival of hosts and vectors in the absence of disease.

Theorem 5. If the hypotheses of Theorem 4 hold and, additionally, $R_{0}<1$ where $R_{0}$ is defined by (3.25), then the disease free equilibrium in which $S_{h}=S_{h}^{0}$ and $S_{v}=S_{v}^{0}$ is locally asymptotically stable to perturbations involving small introductions of disease.

Proof. We prove the theorem by showing that, when $R_{0}<1$, all the roots $\lambda$ of the characteristic equation (3.24) satisfy $\operatorname{Re} \lambda<0$. That establishes that $\left(\tilde{I}_{h}(t), \tilde{I}_{v}(t), \tilde{C}_{v}(t)\right) \rightarrow$ $(0,0,0)$ as $t \rightarrow \infty$. It then follows from the integrals (2.1) and (2.2) that the $E$ variables also approach zero. Having established these facts, as noted earlier the $S_{h}$ and $S_{v}$ equations and their linearisations can then be considered in the case when all other variables are zero, and the previous theorem (Theorem 4) yields that the $S$ variables approach their respective steady state values.

For a contradiction, suppose (3.24) has a root $\lambda^{*}$ such that $\operatorname{Re} \lambda^{*} \geq 0$. We have

$$
\begin{aligned}
\left|\hat{f}_{h}\left(\lambda^{*}+\mu_{h}\right)\right| & =\left|\int_{0}^{\infty} f_{h}(\eta) e^{-\left(\lambda^{*}+\mu_{h}\right) \eta} d \eta\right| \leq \int_{0}^{\infty} f_{h}(\eta) e^{-\left(\operatorname{Re} \lambda^{*}\right) \eta} e^{-\mu_{h} \eta} d \eta \\
& \leq \int_{0}^{\infty} f_{h}(\eta) e^{-\mu_{h} \eta} d \eta
\end{aligned}
$$

so that

$$
\left|\hat{f}_{h}\left(\lambda^{*}+\mu_{h}\right)\right| \leq \hat{f}_{h}\left(\mu_{h}\right)
$$

for $\operatorname{Re} \lambda^{*} \geq 0$. Similarly,

$$
\left|\hat{f}_{v}\left(\lambda^{*}+\mu_{v}+c_{v}\right)\right| \leq \hat{f}_{v}\left(\mu_{v}+c_{v}\right)
$$

Moreover, since $\operatorname{Re} \lambda^{*} \geq 0$,

$$
\left|\lambda^{*}+\mu_{h}+\delta_{h}+\nu\right| \geq \mu_{h}+\delta_{h}+\nu \quad \text { and } \quad\left|\lambda^{*}+\mu_{v}+c_{v}\right| \geq \mu_{v}+c_{v} .
$$


Therefore, any root $\lambda^{*}$ of (3.24) such that $\operatorname{Re} \lambda^{*} \geq 0$ should also satisfy

$$
\left|\lambda^{*}+\mu_{b}\right|=\left|\frac{\beta_{v h} \beta_{h v} c_{v} S_{v}^{0} \hat{f}_{h}\left(\lambda^{*}+\mu_{h}\right) \hat{f}_{v}\left(\lambda^{*}+\mu_{v}+c_{v}\right)}{S_{h}^{0}\left(\lambda^{*}+\mu_{h}+\delta_{h}+\nu\right)\left(\lambda^{*}+\mu_{v}+c_{v}\right)}\right|
$$

which implies

$$
\left|\lambda^{*}+\mu_{b}\right| \leq \frac{\beta_{v h} \beta_{h v} c_{v} S_{v}^{0} \hat{f}_{h}\left(\mu_{h}\right) \hat{f}_{v}\left(\mu_{v}+c_{v}\right)}{S_{h}^{0}\left(\mu_{h}+\delta_{h}+\nu\right)\left(\mu_{v}+c_{v}\right)}=\mu_{b} R_{0}
$$

Hence, $\lambda^{*}$ is in the disk in the complex plane centered at $-\mu_{b}$ of radius $\mu_{b} R_{0}$. Since $R_{0}<1$, this is incompatible with $\operatorname{Re} \lambda^{*} \geq 0$.

Note that $R_{0} \rightarrow 0$ as $c_{v} \rightarrow 0$, suggesting that reducing lice crushing (eg. by not scratching bites) can help to eradicate the disease. Recall that the disease is transmitted

only by crushed lice. Note also that $R_{0}$ depends on $S_{v}^{0}$, which depends on $c_{v}$ through the equation

$$
e^{-\mu_{e} \tau} b_{v}\left(S_{v}^{0}\right)=\left(\mu_{v}+c_{v}\right) S_{v}^{0}
$$

For realistic $b_{v}(\cdot)$, as $c_{v}$ increases $S_{v}^{0}$ decreases. Usually there is no $S_{v}^{0}>0$ when $c_{v}$ is above some finite value. Thus, $R_{0}<1$ both when $c_{v}$ is sufficiently small and also when $c_{v}$ is sufficiently large.

\subsection{Persistence}

The ultimate aim of this section is to establish a set of conditions under which LBRF disease is persistent in the population. These conditions are stated later, in Theorem 6 , and include the requirement that $R_{0}>1$, where $R_{0}$ is defined in (3.25).

\subsubsection{Persistence of lice and humans in the absence of disease}

We start by identifying a minimal set of conditions that will ensure that the susceptible human and lice populations persist in the absence of LBRF disease. In this situation, the equations for $S_{h}(t)$ and $S_{v}(t)$ are

$$
\begin{aligned}
& S_{h}^{\prime}(t)=b_{h}\left(S_{h}(t)\right)-\mu_{h} S_{h}(t) \\
& S_{v}^{\prime}(t)=e^{-\mu_{e} \tau} b_{v}\left(S_{v}(t-\tau)\right)-\left(\mu_{v}+c_{v}\right) S_{v}(t) .
\end{aligned}
$$

Define

$$
\begin{aligned}
R_{0}^{h} & =\frac{1}{\mu_{h}} \liminf _{S \rightarrow 0^{+}} \frac{b_{h}(S)}{S}, \\
R_{0}^{v} & =\frac{1}{\mu_{v}+c_{v}} e^{-\mu_{e} \tau} \liminf _{S \rightarrow 0^{+}} \frac{b_{v}(S)}{S} .
\end{aligned}
$$

The following result establishes persistence of host (human) and vector (louse) populations in the absence of LBRF. 
Proposition 3. Assume that $b_{h}(\cdot)$ and $b_{v}(\cdot)$ are monotone increasing and satisfy conditions (A1) and (A2) and that $R_{0}^{h}>1$ and $R_{0}^{v}>1$. Then, if $S_{h}(0)>0$ and $S_{v}(\theta)>0$ for all $\theta \in[-\tau, 0]$, we have

$$
S_{h \infty}=\liminf _{t \rightarrow \infty} S_{h}(t)>0 \quad \text { and } \quad S_{v \infty}=\liminf _{t \rightarrow \infty} S_{v}(t)>0,
$$

meaning that both host and vector strongly persist.

Proof. We present only the proof that $S_{v \infty}>0$. The proof that $S_{h \infty}>0$ is similar.

Suppose for a contradiction that $\liminf _{t \rightarrow \infty} S_{v}(t)=0$. Then there exists a sequence $t_{n} \rightarrow \infty$ such that $S_{v}\left(t_{n}\right) \rightarrow 0$ as $n \rightarrow \infty$ and $S_{v}^{\prime}\left(t_{n}\right) \leq 0$ for all $n$. Moreover, $t_{n}$ can be chosen such that $S_{v}(t) \geq S_{v}\left(t_{n}\right)$ for all $t \leq t_{n}$, so in particular $S_{v}\left(t_{n}-\tau\right) \geq S_{v}\left(t_{n}\right)$ for all $n$. Since $R_{0}^{v}>1$, we have

$$
\frac{1}{\mu_{v}+c_{v}} e^{-\mu_{e} \tau} \liminf _{S \rightarrow 0^{+}} \frac{b_{v}(S)}{S}>1
$$

and therefore, for sufficiently large $n$,

$$
e^{-\mu_{e} \tau} b_{v}\left(S_{v}\left(t_{n}\right)\right)>\left(\mu_{v}+c_{v}\right) S_{v}\left(t_{n}\right) .
$$

Using that $b_{v}$ is increasing, $S_{v}\left(t_{n}\right) \leq S_{v}\left(t_{n}-\tau\right)$ and (3.29),

$$
e^{-\mu_{e} \tau} b_{v}\left(S_{v}\left(t_{n}\right)\right)-\left(\mu_{v}+c_{v}\right) S_{v}\left(t_{n}\right) \leq e^{-\mu_{e} \tau} b_{v}\left(S_{v}\left(t_{n}-\tau\right)\right)-\left(\mu_{v}+c_{v}\right) S_{v}\left(t_{n}\right)=S_{v}^{\prime}\left(t_{n}\right) \leq 0
$$

which contradicts (3.32).

\subsubsection{Behaviour of susceptible variables at very low levels of disease}

Eventually, we shall prove Theorem 6. Since the proof of that theorem is by a contradiction argument, we first present various results on the properties of solutions of (2.3)-(2.9) in the situation when disease is present at uniformly low levels (in the sense that $\lim \sup _{t \rightarrow \infty} I_{v}(t)<\epsilon$, where $\epsilon$ is a small positive number).

Proposition 4. Suppose that $\epsilon>0$ is a small positive real number such that

$$
\limsup _{t \rightarrow \infty} I_{v}(t)<\epsilon \text {. }
$$

Then,

$$
\limsup _{t \rightarrow \infty} C_{v}(t) \leq \frac{c_{v} \epsilon}{\mu_{b}}, \quad \limsup _{t \rightarrow \infty} I_{h}(t) \leq \frac{\beta_{v h}\left(2 c_{v} \epsilon\right)}{\mu_{b}\left(\mu_{h}+\delta_{h}+\nu\right)}, \quad \limsup _{t \rightarrow \infty} E_{h}(t) \leq \frac{\beta_{v h}\left(2 c_{v} \epsilon\right)}{\mu_{b} \mu_{h}} .
$$

Proof. Let $\epsilon>0$ be a small positive number and suppose that

$$
I_{v}^{\infty}=\limsup _{t \rightarrow \infty} I_{v}(t)<\epsilon .
$$

By the fluctuation method, there exists a sequence $\left(t_{j}\right)$, with $t_{j} \rightarrow \infty$, such that $C_{v}\left(t_{j}\right) \rightarrow$ $C_{v}^{\infty}$ and $C_{v}^{\prime}\left(t_{j}\right) \rightarrow 0$ as $j \rightarrow \infty$, where

$$
C_{v}^{\infty}=\limsup _{t \rightarrow \infty} C_{v}(t) .
$$


Since $\lim \sup _{t \rightarrow \infty} I_{v}(t)<\epsilon$, we have, for $t$ sufficiently large,

$$
I_{v}(t) \leq \epsilon .
$$

From (2.9),

$$
C_{v}^{\prime}\left(t_{j}\right)=c_{v} I_{v}\left(t_{j}\right)-\mu_{b} C_{v}\left(t_{j}\right) \leq c_{v} \epsilon-\mu_{b} C_{v}\left(t_{j}\right) \quad \text { for } j \text { sufficiently large. }
$$

Taking the limit as $j \rightarrow \infty$ gives $0 \leq c_{v} \epsilon-\mu_{b} C_{v}^{\infty}$ and so

$$
C_{v}^{\infty} \leq \frac{c_{v} \epsilon}{\mu_{b}} .
$$

Therefore, if the number of infectious lice is uniformly small, then so is the number of crushed infectious lice. Next we prove that the number of infectious humans also remains uniformly small. Since

$$
C_{v}^{\infty}=\limsup _{t \rightarrow \infty} C_{v}(t) \leq \frac{c_{v} \epsilon}{\mu_{b}},
$$

we have, for sufficiently large $t$, that the following larger bound holds for $C_{v}(t)$ :

$$
C_{v}(t) \leq \frac{2 c_{v} \epsilon}{\mu_{b}} .
$$

Let $T>0$ be large enough so that the estimate (3.33) holds for $t \geq T$, and assume that $t>T$. We estimate the integral term in (2.5) as follows:

$$
\begin{aligned}
& \int_{-\infty}^{t} \beta_{v h} \frac{S_{h}(\xi) C_{v}(\xi)}{N_{h}(\xi)} e^{-\mu_{h}(t-\xi)} f_{h}(t-\xi) d \xi \leq \int_{-\infty}^{t} \beta_{v h} C_{v}(\xi) e^{-\mu_{h}(t-\xi)} f_{h}(t-\xi) d \xi \\
& =\int_{-\infty}^{T} \beta_{v h} C_{v}(\xi) e^{-\mu_{h}(t-\xi)} f_{h}(t-\xi) d \xi+\int_{T}^{t} \beta_{v h} C_{v}(\xi) e^{-\mu_{h}(t-\xi)} f_{h}(t-\xi) d \xi \\
& \leq \int_{-\infty}^{T} \beta_{v h} C_{v}(\xi) e^{-\mu_{h}(t-\xi)} f_{h}(t-\xi) d \xi+\beta_{v h}\left(\frac{2 c_{v} \epsilon}{\mu_{b}}\right) \int_{T}^{t} e^{-\mu_{h}(t-\xi)} f_{h}(t-\xi) d \xi \\
& \leq \beta_{v h} e^{-\mu_{h}(t-T)} \int_{t-T}^{\infty} C_{v}(t-\eta) f_{h}(\eta) d \eta+\beta_{v h}\left(\frac{2 c_{v} \epsilon}{\mu_{b}}\right) \int_{0}^{t-T} e^{-\mu_{h} \eta} f_{h}(\eta) d \eta \\
& \leq \beta_{v h} e^{-\mu_{h}(t-T)}\left(\max _{t \in(-\infty, \infty)} C_{v}(t)\right) \int_{0}^{\infty} f_{h}(\eta) d \eta+\beta_{v h}\left(\frac{2 c_{v} \epsilon}{\mu_{b}}\right) \int_{0}^{\infty} f_{h}(\eta) d \eta \\
& =\beta_{v h} e^{-\mu_{h}(t-T)}\left(\max _{t \in(-\infty, \infty)} C_{v}(t)\right)+\beta_{v h}\left(\frac{2 c_{v} \epsilon}{\mu_{b}}\right)
\end{aligned}
$$

since $\int_{0}^{\infty} f_{h}(\eta) d \eta=1$. Hence

$$
I_{h}^{\prime}(t) \leq \beta_{v h} e^{-\mu_{h}(t-T)}\left(\max _{t \in(-\infty, \infty)} C_{v}(t)\right)+\beta_{v h}\left(\frac{2 c_{v} \epsilon}{\mu_{b}}\right)-\left(\mu_{h}+\delta_{h}+\nu\right) I_{h}(t) .
$$

By the fluctuation method, there exists a sequence $t_{j} \rightarrow \infty$ such that $I_{h}\left(t_{j}\right) \rightarrow I_{h}^{\infty}$ and $I_{h}^{\prime}\left(t_{j}\right) \rightarrow 0$ as $j \rightarrow \infty$, where $I_{h}^{\infty}=\lim \sup _{t \rightarrow \infty} I_{h}(t)$. Evaluating (3.34) at $t=t_{j}$, with $j$ large enough so that $t_{j} \geq T$,

$$
I_{h}^{\prime}\left(t_{j}\right) \leq \beta_{v h} e^{-\mu_{h}\left(t_{j}-T\right)}\left(\max _{t \in(-\infty, \infty)} C_{v}(t)\right)+\beta_{v h}\left(\frac{2 c_{v} \epsilon}{\mu_{b}}\right)-\left(\mu_{h}+\delta_{h}+\nu\right) I_{h}\left(t_{j}\right) .
$$


Letting $j \rightarrow \infty$, and noting that the first term in the above inequality tends to zero, gives

$$
I_{h}^{\infty} \leq \frac{\beta_{v h}\left(2 c_{v} \epsilon\right)}{\mu_{b}\left(\mu_{h}+\delta_{h}+\nu\right)}
$$

Next we show that a similar bound holds for the variable $E_{h}$. From the differential equation for $E_{h}(t)$, equation (2.4),

$$
E_{h}^{\prime}(t) \leq \beta_{v h} \frac{S_{h}(t) C_{v}(t)}{N_{h}(t)}-\mu_{h} E_{h}(t) \leq \beta_{v h} C_{v}(t)-\mu_{h} E_{h}(t)
$$

There is a sequence $t_{j} \rightarrow \infty$ such that $E_{h}\left(t_{j}\right) \rightarrow E_{h}^{\infty}$ and $E_{h}^{\prime}\left(t_{j}\right) \rightarrow 0$ as $j \rightarrow \infty$. From (3.36),

$$
E_{h}^{\prime}\left(t_{j}\right) \leq \beta_{v h} C_{v}\left(t_{j}\right)-\mu_{h} E_{h}\left(t_{j}\right) \leq \beta_{v h}\left(\frac{2 c_{v} \epsilon}{\mu_{b}}\right)-\mu_{h} E_{h}\left(t_{j}\right)
$$

for $j$ sufficiently large, using the bound (3.33). Letting $j \rightarrow \infty$ yields

$$
E_{h}^{\infty} \leq \frac{\beta_{v h}\left(2 c_{v} \epsilon\right)}{\mu_{b} \mu_{h}}
$$

Proposition 5. Suppose $b_{h}(\cdot)$ is increasing and satisfies $(\boldsymbol{A 1})$. Suppose that $\epsilon$ is a small positive real number such that

$$
\limsup _{t \rightarrow \infty} I_{v}(t)<\epsilon
$$

Then

$$
\liminf _{t \rightarrow \infty} S_{h}(t) \geq \bar{S}_{h}^{0}(\epsilon) \quad \text { and } \quad \limsup _{t \rightarrow \infty} S_{h}(t) \leq \hat{S}_{h}^{0}(\epsilon)
$$

where $\bar{S}_{h}^{0}(\epsilon)$ and $\hat{S}_{h}^{0}(\epsilon)$ satisfy

$$
b_{h}\left(\bar{S}_{h}^{0}(\epsilon)\right)=\mu_{h} \bar{S}_{h}^{0}(\epsilon)+\beta_{v h}\left(\frac{2 c_{v} \epsilon}{\mu_{b}}\right),
$$

and

$$
b_{h}\left(\hat{S_{h}^{0}}(\epsilon)+\frac{3 \beta_{v h} c_{v}}{\mu_{b}}\left(\frac{1}{\mu_{h}}+\frac{1}{\mu_{h}+\delta_{h}+\nu}\right) \epsilon\right)+\nu\left(\frac{3 \beta_{v h} c_{v} \epsilon}{\mu_{b}\left(\mu_{h}+\delta_{h}+\nu\right)}\right)=\mu_{h} \hat{S}_{h}^{0}(\epsilon) .
$$

Proof. From the bounds on the limsups of $C_{v}(t), I_{h}(t)$ and $E_{h}(t)$ established in Proposition 4 , the following larger bounds apply for sufficiently large $t$ :

$$
C_{v}(t) \leq \frac{2 c_{v} \epsilon}{\mu_{b}}, \quad I_{h}(t) \leq \frac{3 \beta_{v h} c_{v} \epsilon}{\mu_{b}\left(\mu_{h}+\delta_{h}+\nu\right)}, \quad E_{h}(t) \leq \frac{3 \beta_{v h} c_{v} \epsilon}{\mu_{b} \mu_{h}}
$$

Using that $b_{h}(\cdot)$ is increasing, $I_{h}(t) \geq 0$ and $S_{h}(t) / N_{h}(t) \leq 1$, it follows from (2.3) that

$$
S_{h}^{\prime}(t) \geq b_{h}\left(S_{h}(t)\right)-\beta_{v h}\left(\frac{2 c_{v} \epsilon}{\mu_{b}}\right)-\mu_{h} S_{h}(t) .
$$


It follows that $S_{h}(t) \geq \bar{S}_{h}(t)$, where

$$
\bar{S}_{h}^{\prime}(t)=b_{h}\left(\bar{S}_{h}(t)\right)-\mu_{h} \bar{S}_{h}(t)-\beta_{v h}\left(\frac{2 c_{v} \epsilon}{\mu_{b}}\right) .
$$

Therefore, if $\epsilon$ is sufficiently small then $\bar{S}_{h}(t) \rightarrow \bar{S}_{h}^{0}(\epsilon)$ as $t \rightarrow \infty$, where $\bar{S}_{h}^{0}(\epsilon)$ satisfies (3.37) and has the property that $\bar{S}_{h}^{0}(\epsilon) \rightarrow S_{h}^{0}$ as $\epsilon \rightarrow 0$. By comparison,

$$
\liminf _{t \rightarrow \infty} S_{h}(t) \geq \lim _{t \rightarrow \infty} \bar{S}_{h}(t)=\bar{S}_{h}^{0}(\epsilon) .
$$

Also, from (2.3), and using that $b_{h}(\cdot)$ is increasing,

$$
S_{h}^{\prime}(t) \leq b_{h}\left(S_{h}(t)+\frac{3 \beta_{v h} c_{v} \epsilon}{\mu_{b} \mu_{h}}+\frac{3 \beta_{v h} c_{v} \epsilon}{\mu_{b}\left(\mu_{h}+\delta_{h}+\nu\right)}\right)-\mu_{h} S_{h}(t)+\nu\left(\frac{3 \beta_{v h} c_{v} \epsilon}{\mu_{b}\left(\mu_{h}+\delta_{h}+\nu\right)}\right)
$$

so that, by comparison, $S_{h}(t) \leq \hat{S}_{h}(t)$ where $\hat{S}_{h}(t)$ satisfies the corresponding ordinary differential equation (i.e. $\leq$ replaced by $=$ in the above). Moreover, $\hat{S}_{h}(t) \rightarrow \hat{S}_{h}^{0}(\epsilon)$ as $t \rightarrow \infty$, where $\hat{S}_{h}^{0}(\epsilon)$ satisfies (3.38). Also, $\hat{S}_{h}^{0}(\epsilon) \rightarrow S_{h}^{0}$ as $\epsilon \rightarrow 0$. Moreover,

$$
\limsup _{t \rightarrow \infty} S_{h}(t) \leq \lim _{t \rightarrow \infty} \hat{S}_{h}(t)=\hat{S}_{h}^{0}(\epsilon)
$$

Proposition 5 implies that, for sufficiently large $t$,

$$
\bar{S}_{h}^{0}(\epsilon)-\epsilon \leq S_{h}(t) \leq \hat{S}_{h}^{0}(\epsilon)+\epsilon .
$$

Since the left and right hand sides of the above estimate both approach $S_{h}^{0}$ as $\epsilon \rightarrow 0$, the above estimate establishes that if disease is present at a uniformly low level, in the sense that $\limsup _{t \rightarrow \infty} I_{v}(t)<\epsilon$, the number of susceptible humans $S_{h}(t)$ remains close to its steady state value $S_{h}^{0}$ in the absence of disease.

Next we establish an upper bound for $S_{v}(t)$ that holds under all circumstances (not only when $I_{h}(t)$ is small).

Proposition 6. Suppose that $b_{v}$ is a positive bounded function. Then

$$
\limsup _{t \rightarrow \infty} S_{v}(t) \leq \frac{e^{-\mu_{e} \tau} b_{v}^{\text {sup }}}{\mu_{v}+c_{v}}
$$

where $b_{v}^{\text {sup }}=\sup _{S \geq 0} b_{v}(S)$.

Proof. From (2.6), $S_{v}^{\prime}(t) \leq e^{-\mu_{e} \tau} b_{v}^{\text {sup }}-\left(\mu_{v}+c_{v}\right) S_{v}(t)$ and (3.39) follows.

Next, we establish an upper bound for $E_{v}(t)$ that applies when $I_{v}(t)$ remains small. This bound is similar to those of Proposition 4 , but its derivation requires the additional assumption that the function $b_{v}$ should be bounded. 
Proposition 7. Suppose that $\epsilon>0$ is a small number such that

$$
\limsup _{t \rightarrow \infty} I_{v}(t)<\epsilon
$$

and suppose that $b_{v}(\cdot)$ is a bounded function, and that $b_{h}(\cdot)$ is increasing and satisfies (A1). Then

$$
\limsup _{t \rightarrow \infty} E_{v}(t) \leq \frac{\beta_{h v}}{\bar{S}_{h}^{0}(\epsilon)-\epsilon}\left(\frac{2 e^{-\mu_{e} \tau} b_{v}^{\text {sup }}}{\left(\mu_{v}+c_{v}\right)^{2}}\right)\left(\frac{3 \beta_{v h} c_{v} \epsilon}{\mu_{b}\left(\mu_{h}+\delta_{h}+\nu\right)}\right),
$$

for $\epsilon$ sufficiently small such that $\bar{S}_{h}^{0}(\epsilon)-\epsilon>0$.

Proof. Since the hypotheses include those of Proposition 4, we may use the upper bound for $\lim \sup _{t \rightarrow \infty} I_{h}(t)$ from Proposition 4 , and that for $\lim \sup _{t \rightarrow \infty} S_{v}(t)$ from Proposition 6 . From (2.7), for $t$ sufficiently large we have

$$
E_{v}^{\prime}(t) \leq \frac{\beta_{h v}}{N_{h}(t)}\left(\frac{2 e^{-\mu_{e} \tau} b_{v}^{\text {sup }}}{\mu_{v}+c_{v}}\right)\left(\frac{3 \beta_{v h} c_{v} \epsilon}{\mu_{b}\left(\mu_{h}+\delta_{h}+\nu\right)}\right)-\left(\mu_{v}+c_{v}\right) E_{v}(t)
$$

But $N_{h}(t)=S_{h}(t)+E_{h}(t)+I_{h}(t) \geq S_{h}(t) \geq \bar{S}_{h}^{0}(\epsilon)-\epsilon$ for $t$ sufficiently large, so that

$$
E_{v}^{\prime}(t) \leq \frac{\beta_{h v}}{\bar{S}_{h}^{0}(\epsilon)-\epsilon}\left(\frac{2 e^{-\mu_{e} \tau} b_{v}^{\text {sup }}}{\mu_{v}+c_{v}}\right)\left(\frac{3 \beta_{v h} c_{v} \epsilon}{\mu_{b}\left(\mu_{h}+\delta_{h}+\nu\right)}\right)-\left(\mu_{v}+c_{v}\right) E_{v}(t)
$$

and therefore (3.40) holds.

Next we show that, when the $I_{v}, I_{h}$ and $C_{v}$ variables remain small, $S_{v}(t)$ remains close to its steady state value.

Proposition 8. Suppose that $b_{v}(\cdot)$ is increasing, bounded above and satisfies (A2) and that $b_{h}(\cdot)$ is increasing and satisfies $(\boldsymbol{A} \mathbf{1})$. Let $\epsilon>0$ be a small number such that

$$
\limsup _{t \rightarrow \infty} I_{v}(t)<\epsilon .
$$

Then $S_{v}(t)$ remains close to its steady state value $S_{v}^{0}$ in the sense that

$$
\bar{S}_{v}^{0}(\epsilon) \leq \liminf _{t \rightarrow \infty} S_{v}(t) \leq \limsup _{t \rightarrow \infty} S_{v}(t) \leq \hat{S}_{v}^{0}(\epsilon)
$$

where $\hat{S}_{v}^{0}(\epsilon)$ and $\bar{S}_{v}^{0}(\epsilon)$ respectively satisfy

$$
\begin{array}{r}
e^{-\mu_{e} \tau} b_{v}\left(\hat{S}_{v}^{0}(\epsilon)+\frac{\beta_{h v}}{\overline{S_{h}^{0}}(\epsilon)-\epsilon}\left(\frac{2 e^{-\mu_{e} \tau} b_{v}^{\text {sup }}}{\left(\mu_{v}+c_{v}\right)^{2}}\right)\left(\frac{3 \beta_{v h} c_{v} \epsilon}{\mu_{b}\left(\mu_{h}+\delta_{h}+\nu\right)}\right)+\epsilon\right)=\left(\mu_{v}+c_{v}\right) \hat{S}_{v}^{0}(\epsilon), \\
e^{-\mu_{e} \tau} b_{v}\left(\bar{S}_{v}^{0}(\epsilon)\right)=\frac{\beta_{h v}}{\bar{S}_{h}^{0}(\epsilon)-\epsilon}\left(\frac{2 e^{-\mu_{e} \tau} b_{v}^{\text {sup }}}{\mu_{v}+c_{v}}\right)\left(\frac{3 \beta_{v h} c_{v} \epsilon}{\mu_{b}\left(\mu_{h}+\delta_{h}+\nu\right)}\right)+\left(\mu_{v}+c_{v}\right) \bar{S}_{v}^{0}(\epsilon) .
\end{array}
$$

Note that $\hat{S}_{v}^{0}(\epsilon)$ and $\bar{S}_{v}^{0}(\epsilon)$ both approach $S_{v}^{0}$ as $\epsilon \rightarrow 0$, where $S_{v}^{0}$ is given by (3.18). 
Proof. For $t$ sufficiently large,

$$
I_{v}(t) \leq \epsilon \quad \text { and } \quad E_{v}(t) \leq \frac{\beta_{h v}}{S_{h}^{0}(\epsilon)-\epsilon}\left(\frac{2 e^{-\mu_{e} \tau} b_{v}^{\text {sup }}}{\left(\mu_{v}+c_{v}\right)^{2}}\right)\left(\frac{3 \beta_{v h} c_{v} \epsilon}{\mu_{b}\left(\mu_{h}+\delta_{h}+\nu\right)}\right) .
$$

Therefore, for $t$ sufficiently large,

$S_{v}^{\prime}(t) \leq e^{-\mu_{e} \tau} b_{v}\left(S_{v}(t-\tau)+\frac{\beta_{h v}}{S_{h}^{0}(\epsilon)-\epsilon}\left(\frac{2 e^{-\mu_{e} \tau} b_{v}^{\text {sup }}}{\left(\mu_{v}+c_{v}\right)^{2}}\right)\left(\frac{3 \beta_{v h} c_{v} \epsilon}{\mu_{b}\left(\mu_{h}+\delta_{h}+\nu\right)}\right)+\epsilon\right)-\left(\mu_{v}+c_{v}\right) S_{v}(t)$.

By the comparison theorems in Smith [15] and Kuang [10], since $b_{v}(\cdot)$ is increasing, $S_{v}(t) \leq \hat{S}_{v}(t)$ where

${\hat{S_{v}}}^{\prime}(t)=e^{-\mu_{e} \tau} b_{v}\left(\hat{S}_{v}(t-\tau)+\frac{\beta_{h v}}{\bar{S}_{h}^{0}(\epsilon)-\epsilon}\left(\frac{2 e^{-\mu_{e} \tau} b_{v}^{\mathrm{sup}}}{\left(\mu_{v}+c_{v}\right)^{2}}\right)\left(\frac{3 \beta_{v h} c_{v} \epsilon}{\mu_{b}\left(\mu_{h}+\delta_{h}+\nu\right)}\right)+\epsilon\right)-\left(\mu_{v}+c_{v}\right) \hat{S}_{v}(\epsilon)$

and, again using that $b_{v}(\cdot)$ is increasing, $\hat{S}_{v}(t)$ generically converges as $t \rightarrow \infty$ to the unique positive steady state $\hat{S}_{v}^{0}(\epsilon)$ which satisfies the first equation of (3.41). Moreover, $\hat{S_{v}^{0}}(\epsilon) \rightarrow S_{v}^{0}$ as $\epsilon \rightarrow 0$ and

$$
\limsup _{t \rightarrow \infty} S_{v}(t) \leq \lim _{t \rightarrow \infty} \hat{S}_{v}(t)=\hat{S}_{v}^{0}(\epsilon) .
$$

Since $b_{v}(\cdot)$ is increasing, for $t$ sufficiently large, using that $N_{h}(t) \geq S_{h}(t) \geq \bar{S}_{h}^{0}(\epsilon)-\epsilon$,

$$
\begin{aligned}
S_{v}^{\prime}(t) & \geq e^{-\mu_{e} \tau} b_{v}\left(S_{v}(t-\tau)\right)-\beta_{h v} \frac{S_{v}(t)}{N_{h}(t)}\left(\frac{3 \beta_{v h} c_{v} \epsilon}{\mu_{b}\left(\mu_{h}+\delta_{h}+\nu\right)}\right)-\left(\mu_{v}+c_{v}\right) S_{v}(t) \\
& \geq e^{-\mu_{e} \tau} b_{v}\left(S_{v}(t-\tau)\right)-\frac{\beta_{h v}}{\overline{S_{h}^{0}}(\epsilon)-\epsilon}\left(\frac{2 e^{-\mu_{e} \tau} b_{v}^{\text {sup }}}{\mu_{v}+c_{v}}\right)\left(\frac{3 \beta_{v h} c_{v} \epsilon}{\mu_{b}\left(\mu_{h}+\delta_{h}+\nu\right)}\right)-\left(\mu_{v}+c_{v}\right) S_{v}(t) .
\end{aligned}
$$

Therefore, $S_{v}(t) \geq \bar{S}_{v}(t)$ where $\bar{S}_{v}(t)$ satisfies the ordinary differential equation associated with the above differential inequality (when we replace $\geq$ by $=$ ), and therefore

$$
\liminf _{t \rightarrow \infty} S_{v}(t) \geq \lim _{t \rightarrow \infty} \bar{S}_{v}(t)=\bar{S}_{v}^{0}(\epsilon)
$$

where $\bar{S}_{v}^{0}(\epsilon)$ satisfies the second equation of (3.41). Note that, as $\epsilon \rightarrow 0,{\overline{S_{v}^{0}}}^{0}(\epsilon) \rightarrow S_{v}^{0}$, the equilibrium value of $S_{v}$ in the case of no disease.

Finally, we conclude that, for sufficiently large $t$,

$$
\overline{S_{v}^{0}}(\epsilon)-\epsilon \leq S_{v}(t) \leq \hat{S}_{v}^{0}(\epsilon)+\epsilon .
$$

Therefore, $S_{v}(t)$ remains near to $S_{v}^{0}$ when disease is present at a low level.

\subsubsection{Persistence of disease}

With the benefit of the above results, we may present our main result of this section: 
Theorem 6. Assume that $b_{h}(\cdot)$ and $b_{v}(\cdot)$ are increasing functions satisfying $(\boldsymbol{A} \mathbf{1})$ and (A2), respectively. Additionally, assume that $b_{v}$ is bounded above. If $R_{0}>1, R_{0}^{h}>1$ and $R_{0}^{v}>1$, where $R_{0}$ is defined in (3.25), and $R_{0}^{h}$ and $R_{0}^{v}$ are defined in (3.30) and (3.31), respectively, then there exists $\epsilon>0$ such that

$$
\limsup _{t \rightarrow \infty} I_{v}(t) \geq \epsilon,
$$

for all solutions with $S_{h}(0)>0, I_{h}(0)>0, I_{v}(0)>0, C_{v}(0)>0$ and $S_{v}(t)>0$ for some $t \in[-\tau, 0]$.

Proof. If the statement is not true, then for any $\epsilon>0$ there is a solution such that $I_{v}(0)>0$ and

$$
\limsup _{t \rightarrow \infty} I_{v}(t)<\epsilon,
$$

and the results of the previous propositions hold. By shifting forward in time, we have

$$
0<I_{v}(t) \leq \epsilon, \quad E_{h}(t) \leq c_{1} \epsilon, \quad I_{h}(t) \leq c_{2} \epsilon,
$$

where

$$
c_{1}=\frac{3 \beta_{v h} c_{v}}{\mu_{b} \mu_{h}}, \quad c_{2}=\frac{3 \beta_{v h} c_{v}}{\mu_{b}\left(\mu_{h}+\delta_{h}+\nu\right)} .
$$

Also, for $t$ sufficiently large we know from Propositions 5 and 8 that

$$
\bar{S}_{h}^{0}(\epsilon)-\epsilon \leq S_{h}(t) \leq \hat{S}_{h}^{0}(\epsilon)+\epsilon, \quad \bar{S}_{v}^{0}(\epsilon)-\epsilon \leq S_{v}(t) \leq \hat{S}_{v}^{0}(\epsilon)+\epsilon .
$$

Since $N_{h}(t)=S_{h}(t)+E_{h}(t)+I_{h}(t)$, we have $N_{h}(t) \leq \hat{S}_{h}^{0}(\epsilon)+\epsilon+c_{1} \epsilon+c_{2} \epsilon$. Using these inequalities in equations (2.5) and (2.8) of the model, we obtain

$$
\begin{aligned}
& I_{h}^{\prime}(t) \geq \int_{0}^{t} \beta_{v h} \frac{\left(\bar{S}_{h}^{0}(\epsilon)-\epsilon\right) C_{v}(\xi)}{\hat{S}_{h}^{0}(\epsilon)+\epsilon+c_{1} \epsilon+c_{2} \epsilon} e^{-\mu_{h}(t-\xi)} f_{h}(t-\xi) d \xi-\left(\mu_{h}+\delta_{h}+\nu\right) I_{h}(t), \\
& I_{v}^{\prime}(t) \geq \int_{0}^{t} \beta_{h v} \frac{\left(\overline{S_{v}^{0}}(\epsilon)-\epsilon\right) I_{h}(\xi)}{\hat{S}_{h}^{0}(\epsilon)+\epsilon+c_{1} \epsilon+c_{2} \epsilon} e^{-\left(\mu_{v}+c_{v}\right)(t-\xi)} f_{v}(t-\xi) d \xi-\left(\mu_{v}+c_{v}\right) I_{v}(t),
\end{aligned}
$$

where additionally we have replaced the lower limit $-\infty$ on the integrals by zero, since we may discard the non-negative contribution of $\int_{-\infty}^{0}$. The Laplace transform can be applied to inequalities as long as the transform variable is real. Therefore, applying the Laplace transform operator, denoted by $\mathcal{L}$, and restricting to real values of the transform variable $\lambda$, we obtain

$\lambda \mathcal{L}\left\{I_{h}\right\}(\lambda)-I_{h}(0) \geq \beta_{v h} \frac{\bar{S}_{h}^{0}(\epsilon)-\epsilon}{\hat{S}_{h}^{0}(\epsilon)+\epsilon+c_{1} \epsilon+c_{2} \epsilon} \mathcal{L}\left\{C_{v}\right\}(\lambda) \mathcal{L}\left\{e^{-\mu_{h} t} f_{h}(t)\right\}-\left(\mu_{h}+\delta_{h}+\nu\right) \mathcal{L}\left\{I_{h}\right\}(\lambda)$,

where we have used the convolution theorem for the Laplace transform. Since

$$
\mathcal{L}\left\{e^{-\mu_{h} t} f_{h}(t)\right\}=\mathcal{L}\left\{f_{h}\right\}\left(\lambda+\mu_{h}\right),
$$


further simplification, ignoring the non-negative term $I_{h}(0)$, leads to

$$
\mathcal{L}\left\{I_{h}\right\}(\lambda) \geq \frac{1}{\lambda+\mu_{h}+\delta_{h}+\nu}\left(\frac{\beta_{v h}\left(\bar{S}_{h}^{0}(\epsilon)-\epsilon\right)}{\hat{S}_{h}^{0}(\epsilon)+\epsilon+c_{1} \epsilon+c_{2} \epsilon}\right) \mathcal{L}\left\{C_{v}\right\}(\lambda) \mathcal{L}\left\{f_{h}\right\}\left(\lambda+\mu_{h}\right) .
$$

Similarly,

$$
\begin{aligned}
\mathcal{L}\left\{I_{v}\right\}(\lambda) \geq \frac{1}{\lambda+\mu_{v}+c_{v}}\left(\frac{\beta_{h v}\left(\bar{S}_{v}^{0}(\epsilon)-\epsilon\right)}{\hat{S}_{h}^{0}(\epsilon)+\epsilon+c_{1} \epsilon+c_{2} \epsilon}\right) \mathcal{L}\left\{I_{h}\right\}(\lambda) \mathcal{L}\left\{f_{v}\right\}\left(\lambda+\mu_{v}+c_{v}\right), \\
\mathcal{L}\left\{C_{v}\right\}(\lambda)=\frac{1}{\lambda+\mu_{b}} c_{v} \mathcal{L}\left\{I_{v}\right\}(\lambda) .
\end{aligned}
$$

Multiplying these inequalities and simplifying,

$$
\begin{aligned}
1 \geq & \frac{1}{\lambda+\mu_{v}+c_{v}}\left(\frac{\beta_{h v}\left(\bar{S}_{v}^{0}(\epsilon)-\epsilon\right)}{\hat{S_{h}^{0}}(\epsilon)+\epsilon+c_{1} \epsilon+c_{2} \epsilon}\right) \mathcal{L}\left\{f_{v}\right\}\left(\lambda+\mu_{v}+c_{v}\right) \frac{1}{\lambda+\mu_{h}+\delta_{h}+\nu} \\
& \times\left(\frac{\beta_{v h}\left(\bar{S}_{h}^{0}(\epsilon)-\epsilon\right)}{\hat{S_{h}^{0}}(\epsilon)+\epsilon+c_{1} \epsilon+c_{2} \epsilon}\right) \mathcal{L}\left\{f_{h}\right\}\left(\lambda+\mu_{h}\right) \frac{1}{\lambda+\mu_{b}} c_{v} .
\end{aligned}
$$

Letting $\lambda \rightarrow 0$ and then $\epsilon \rightarrow 0$, and recalling that $\hat{S}_{h}^{0}(\epsilon) \rightarrow S_{h}^{0}, \bar{S}_{h}^{0}(\epsilon) \rightarrow S_{h}^{0}$ and $\bar{S}_{v}^{0}(\epsilon) \rightarrow$ $S_{v}^{0}$ as $\epsilon \rightarrow 0$, the above inequality takes the form

$$
1 \geq \frac{\beta_{v h} \beta_{h v} c_{v} S_{v}^{0} \mathcal{L}\left\{f_{h}\right\}\left(\mu_{h}\right) \mathcal{L}\left\{f_{v}\right\}\left(\mu_{v}+c_{v}\right)}{\left(\mu_{h}+\delta_{h}+\nu\right)\left(\mu_{v}+c_{v}\right) \mu_{b} S_{h}^{0}}=R_{0},
$$

which contradicts the assumption that $R_{0}>1$.

\subsection{Eradication of disease: the nonlinear regime}

In this section we obtain a condition, stronger than that of Theorem 5 , that is sufficient to establish that the $I_{h}, I_{v}$ and $C_{v}$ variables approach zero as components of the solution of the full nonlinear model (2.3)-(2.9). There is no linearisation in this result, even though linear theory features. What happens is that we use comparison theorems to bound the $I_{h}, I_{v}$ and $C_{v}$ components of the solution by other functions that satisfy a linear system. In the case of (2.8), handling the denominator term $N_{h}(\xi)$ presents difficulties due to the need to retain the $I_{h}(\xi)$ factor in the integrand for the generation of a linear system suitable for the application of comparison theory. For (2.5) there is no such problem, $C_{v}(\xi)$ is the factor to be retained and so we simply use $S_{h} / N_{h} \leq 1$. To deal with the difficulties with (2.8) we need to impose a smallness restriction on the initial numbers of infectious individuals.

Theorem 7. Suppose the birth functions $b_{h}$ and $b_{v}$ satisfy $(\boldsymbol{A} \mathbf{1})$ and (A2), respectively, and that both are monotone nondecreasing on $\left[0, S_{h}^{0}\right]$ and $\left[0, S_{v}^{0}\right]$, respectively, with $b_{v}$ bounded. Assume also that

$$
\frac{c_{v} \beta_{v h} \beta_{h v}\left(\frac{e^{-\mu_{e} \tau} b_{v}^{\text {sup }}}{\mu_{v}+c_{v}}\right) \mathcal{L}\left\{f_{h}\right\}\left(\mu_{h}\right) \mathcal{L}\left\{f_{v}\right\}\left(\mu_{v}+c_{v}\right)}{S_{h}^{0}\left(\mu_{h}+\delta_{h}+\nu\right)\left(\mu_{v}+c_{v}\right) \mu_{b}}<1
$$

where $\mathcal{L}$ denotes the Laplace transform operator. Then, provided that 
(i) the variables $I_{h}(t), I_{v}(t)$ and $C_{v}(t)$ are sufficiently small initially;

(ii) the total human population $N_{h}(t)$ is initially not too far below its disease-free equilibrium value $S_{h}^{0}$;

(iii) $S_{v}(0) \leq e^{-\mu_{e} \tau} b_{v}^{\text {sup }} /\left(\mu_{v}+c_{v}\right)$,

then, as components of the solution of the full nonlinear model (2.3)-(2.9), the variables $\left(I_{h}(t), I_{v}(t), C_{v}(t)\right) \rightarrow(0,0,0)$ as $t \rightarrow \infty$.

Proof. We begin by defining, indirectly, a certain continuous function $\phi(\epsilon)$ that satisfies $\phi(0)=0$. We know from (A1) that $x=S_{h}^{0}$ is the unique positive root of the equation $b_{h}(x)=\mu_{h} x$. If the right hand side of this equation is changed to $\mu_{h} x+\delta_{h} k \epsilon$ for sufficiently small positive $\epsilon$, then its root drops from $S_{h}^{0}$ to a smaller value that we call $S_{h}^{0}-\phi(\epsilon)$. In this way, we implicitly define a function $\phi(\epsilon)$, and moreover $\phi(0)=0$. With $\phi(\epsilon)$ thus defined, let $\epsilon>0$ be sufficiently small such that

$$
\frac{c_{v} \beta_{v h} \beta_{h v}\left(\frac{e^{-\mu_{e} \tau} b_{v}^{\text {sup }}}{\mu_{v}+c_{v}}\right) \mathcal{L}\left\{f_{h}\right\}\left(\mu_{h}\right) \mathcal{L}\left\{f_{v}\right\}\left(\mu_{v}+c_{v}\right)}{\left(S_{h}^{0}-\phi(\epsilon)\right)\left(\mu_{h}+\delta_{h}+\nu\right)\left(\mu_{v}+c_{v}\right) \mu_{b}}<1 .
$$

Recall that the total number of humans $N_{h}(t)=S_{h}(t)+E_{h}(t)+I_{h}(t)$ satisfies

$$
N_{h}^{\prime}(t)=b_{h}\left(N_{h}(t)\right)-\mu_{h} N_{h}(t)-\delta_{h} I_{h}(t) .
$$

Suppose that the initial data is such that

$$
I_{h}(t) \leq \epsilon \quad \text { for all } t \leq 0, \quad \text { and } \quad S_{h}(0)+E_{h}(0)+I_{h}(0) \geq S_{h}^{0}-\phi(\epsilon),
$$

and suppose that a constant $k$ can be found such that $I_{h}(t) \leq k \epsilon$ for all $t>0$ (this will be confirmed later, in Proposition 9). Using the bound $I_{h}(t) \leq k \epsilon$,

$$
N_{h}^{\prime}(t) \geq b_{h}\left(N_{h}(t)\right)-\mu_{h} N_{h}(t)-\delta_{h} k \epsilon .
$$

A simple comparison argument together with elementary properties of solutions of onedimensional ODEs, and the above remarks concerning the definition of $\phi(\epsilon)$, yields that

$$
N_{h}(t) \geq \min \left(S_{h}(0)+E_{h}(0)+I_{h}(0), S_{h}^{0}-\phi(\epsilon)\right)=S_{h}^{0}-\phi(\epsilon) \quad \text { for all } t \geq 0 .
$$

From the proof of Proposition 6 it is clear that our assumption $S_{v}(0) \leq e^{-\mu_{e} \tau} b_{v}^{\text {sup }} /\left(\mu_{v}+c_{v}\right)$ implies that

$$
S_{v}(t) \leq \frac{e^{-\mu_{e} \tau} b_{v}^{\text {sup }}}{\mu_{v}+c_{v}} \quad \text { for all } t \geq 0,
$$

where $b_{v}^{\text {sup }}=\sup _{S \geq 0} b_{v}(S)$. Using this information and that $S_{h} / N_{h} \leq 1$, we obtain from (2.5) and (2.8) the following system of differential equations and inequalities for $I_{h}, I_{v}$ and $C_{v}$, holding for all $t \geq 0$ :

$$
\begin{aligned}
I_{h}^{\prime}(t) & \leq \int_{-\infty}^{t} \beta_{v h} C_{v}(\xi) e^{-\mu_{h}(t-\xi)} f_{h}(t-\xi) d \xi-\left(\mu_{h}+\delta_{h}+\nu\right) I_{h}(t) \\
I_{v}^{\prime}(t) & \leq \int_{-\infty}^{t} \beta_{h v} \frac{\left(\frac{e^{-\mu_{e} \tau} b_{v}^{\text {sup }}}{\mu_{v}+c_{v}}\right)}{S_{h}^{0}-\phi(\epsilon)} I_{h}(\xi) e^{-\left(\mu_{v}+c_{v}\right)(t-\xi)} f_{v}(t-\xi) d \xi-\left(\mu_{v}+c_{v}\right) I_{v}(t) \\
C_{v}^{\prime}(t) & =c_{v} I_{v}(t)-\mu_{b} C_{v}(t) .
\end{aligned}
$$


By a comparison argument, $I_{h} \leq \bar{I}_{h}, I_{v} \leq \bar{I}_{v}$ and $C_{v} \leq \bar{C}_{v}$ where

$$
\begin{aligned}
\bar{I}_{h}^{\prime}(t) & =\int_{-\infty}^{t} \beta_{v h} \bar{C}_{v}(\xi) e^{-\mu_{h}(t-\xi)} f_{h}(t-\xi) d \xi-\left(\mu_{h}+\delta_{h}+\nu\right) \bar{I}_{h}(t) \\
\bar{I}_{v}^{\prime}(t) & =\int_{-\infty}^{t} \beta_{h v} \frac{\left(\frac{e^{-\mu_{e} \tau} b_{v}^{\text {sup }}}{\mu_{v}+c_{v}}\right)}{S_{h}^{0}-\phi(\epsilon)} \bar{I}_{h}(\xi) e^{-\left(\mu_{v}+c_{v}\right)(t-\xi)} f_{v}(t-\xi) d \xi-\left(\mu_{v}+c_{v}\right) \bar{I}_{v}(t) \\
\bar{C}_{v}^{\prime}(t) & =c_{v} \bar{I}_{v}(t)-\mu_{b} \bar{C}_{v}(t) .
\end{aligned}
$$

We have bounded the solution components $I_{h}, I_{v}$ and $C_{v}$ by new variables that satisfy the above linear system. A search for non-trivial solutions of the form $\left(\bar{I}_{h}, \bar{I}_{v}, \bar{C}_{v}\right)=$ $e^{\lambda t}\left(c_{1}, c_{2}, c_{3}\right)$ yields the characteristic equation

$$
\left(\lambda+\mu_{h}+\delta_{h}+\nu\right)\left(\lambda+\mu_{v}+c_{v}\right)\left(\lambda+\mu_{b}\right)=\frac{c_{v} \beta_{v h} \beta_{h v} e^{-\mu_{e} \tau} b_{v}^{\sup }}{\left(\mu_{v}+c_{v}\right)\left(S_{h}^{0}-\phi(\epsilon)\right)} \mathcal{L}\left\{f_{h}\right\}\left(\lambda+\mu_{h}\right) \mathcal{L}\left\{f_{v}\right\}\left(\lambda+\mu_{v}+c_{v}\right)
$$

where $\mathcal{L}$ denotes Laplace transform. Analysis similar to that presented in the proof of Theorem 5 yields that, if

$$
\frac{c_{v} \beta_{v h} \beta_{h v}\left(\frac{e^{-\mu_{e} \tau} b_{v}^{\text {sup }}}{\mu_{v}+c_{v}}\right) \mathcal{L}\left\{f_{h}\right\}\left(\mu_{h}\right) \mathcal{L}\left\{f_{v}\right\}\left(\mu_{v}+c_{v}\right)}{\left(S_{h}^{0}-\phi(\epsilon)\right)\left(\mu_{h}+\delta_{h}+\nu\right)\left(\mu_{v}+c_{v}\right) \mu_{b}}<1
$$

which holds (see early in the proof), then all roots $\lambda$ of the characteristic equation (3.44) satisfy $\operatorname{Re} \lambda<0$ so that $\left(\bar{I}_{h}(t), \bar{I}_{v}(t), \bar{C}_{v}(t)\right) \rightarrow(0,0,0)$ as $t \rightarrow \infty$. Hence also $\left(I_{h}(t), I_{v}(t), C_{v}(t)\right) \rightarrow$ $(0,0,0)$ as $t \rightarrow \infty$.

The following proposition confirms that if the three variables $I_{h}, I_{v}$ and $C_{v}$ start small then they remain small for all $t>0$.

Proposition 9. Suppose the hypotheses of Theorem 7 hold and that the initial data is such that

$$
0 \leq I_{h}(t), I_{v}(t), C_{v}(t) \leq \epsilon \quad \text { for all } t \leq 0 .
$$

Then there exists $k>0$ such that $I_{h}(t) \leq k \epsilon$ for all $t>0$. Furthermore, $k$ is independent of the initial data, and is also independent of $\epsilon$ for $\epsilon>0$ sufficiently small.

Proof. Let

$$
K=\frac{\beta_{h v} e^{-\mu_{e} \tau} b_{v}^{\sup }}{\left(\mu_{v}+c_{v}\right)\left(S_{h}^{0}-\phi(\epsilon)\right)}
$$

with the function $\phi(\epsilon)$ defined early in the proof of Theorem 7, and $\epsilon$ again assumed to be sufficiently small so that (3.43) holds. Then the differential equations for $\bar{I}_{h}(t), \bar{I}_{v}(t)$ and $\bar{C}_{v}(t)$ that feature in the proof of Theorem 7 can be written as

$$
\begin{aligned}
\bar{I}_{h}^{\prime}(t) & =\int_{-\infty}^{t} \beta_{v h} \bar{C}_{v}(\xi) e^{-\mu_{h}(t-\xi)} f_{h}(t-\xi) d \xi-\left(\mu_{h}+\delta_{h}+\nu\right) \bar{I}_{h}(t), \\
\bar{I}_{v}^{\prime}(t) & =K \int_{-\infty}^{t} \bar{I}_{h}(\xi) e^{-\left(\mu_{v}+c_{v}\right)(t-\xi)} f_{v}(t-\xi) d \xi-\left(\mu_{v}+c_{v}\right) \bar{I}_{v}(t), \\
\bar{C}_{v}^{\prime}(t) & =c_{v} \bar{I}_{v}(t)-\mu_{b} \bar{C}_{v}(t) .
\end{aligned}
$$


We take the Laplace transform of each equation, splitting the integral as $\int_{-\infty}^{0}+\int_{0}^{t}$ and applying the convolution theorem to the second integral. With $\mathcal{L}$ denoting the Laplace transform operator, and $\lambda$ the transform variable, we obtain

$$
\begin{aligned}
\lambda \mathcal{L}\left\{\bar{I}_{h}\right\}(\lambda)-\bar{I}_{h}(0)= & \beta_{v h} \mathcal{L}\left\{\int_{-\infty}^{0} \bar{C}_{v}(\xi) e^{-\mu_{h}(t-\xi)} f_{h}(t-\xi) d \xi\right\}+\beta_{v h} \mathcal{L}\left\{\bar{C}_{v}\right\}(\lambda) \mathcal{L}\left\{f_{h}\right\}\left(\lambda+\mu_{h}\right) \\
& -\left(\mu_{h}+\delta_{h}+\nu\right) \mathcal{L}\left\{\bar{I}_{h}\right\}(\lambda)
\end{aligned}
$$

and two similar equations, so that

$$
\begin{gathered}
\left(\begin{array}{ccc}
\lambda+\mu_{h}+\delta_{h}+\nu & 0 & -\beta_{v h} \mathcal{L}\left\{f_{h}\right\}\left(\lambda+\mu_{h}\right) \\
-K \mathcal{L}\left\{f_{v}\right\}\left(\lambda+\mu_{v}+c_{v}\right) & \lambda+\mu_{v}+c_{v} & 0 \\
0 & -c_{v} & \lambda+\mu_{b}
\end{array}\right)\left(\begin{array}{c}
\mathcal{L}\left\{\bar{I}_{h}\right\}(\lambda) \\
\mathcal{L}\left\{\bar{I}_{v}\right\}(\lambda) \\
\mathcal{L}\left\{\bar{C}_{v}\right\}(\lambda)
\end{array}\right) \\
=\left(\begin{array}{c}
\bar{I}_{h}(0)+\beta_{v h} \int_{0}^{\infty} e^{-\lambda \bar{t}} \int_{-\infty}^{0} \bar{C}_{v}(\xi) e^{-\mu_{h}(\bar{t}-\xi)} f_{h}(\bar{t}-\xi) d \xi d \bar{t} \\
\bar{I}_{v}(0)+K \int_{0}^{\infty} e^{-\lambda \bar{t}} \int_{-\infty}^{0} \bar{I}_{h}(\xi) e^{-\left(\mu_{v}+c_{v}\right)(\bar{t}-\xi)} f_{v}(\bar{t}-\xi) d \xi d \bar{t} \\
\bar{C}_{v}(0)
\end{array}\right)
\end{gathered}
$$

Let $A(\lambda)$ denote the matrix in the left hand side. Then

$$
\left(\begin{array}{c}
\mathcal{L}\left\{\bar{I}_{h}\right\} \\
\mathcal{L}\left\{\bar{I}_{v}\right\} \\
\mathcal{L}\left\{\bar{C}_{v}\right\}
\end{array}\right)=\frac{1}{\Delta(\lambda)}\left(A_{j k}(\lambda)\right)^{T}\left(\begin{array}{c}
\bar{I}_{h}(0)+\beta_{v h} \int_{0}^{\infty} e^{-\lambda \bar{t}} \int_{-\infty}^{0} \bar{C}_{v}(\xi) e^{-\mu_{h}(\bar{t}-\xi)} f_{h}(\bar{t}-\xi) d \xi d \bar{t} \\
\bar{I}_{v}(0)+K \int_{0}^{\infty} e^{-\lambda \bar{t}} \int_{-\infty}^{0} \bar{I}_{h}(\xi) e^{-\left(\mu_{v}+c_{v}\right)(\bar{t}-\xi)} f_{v}(\bar{t}-\xi) d \xi d \bar{t} \\
\bar{C}_{v}(0)
\end{array}\right)
$$

where $\Delta(\lambda)$ is the determinant of $A(\lambda)$, given by

$\Delta(\lambda)=\left(\lambda+\mu_{h}+\delta_{h}+\nu\right)\left(\lambda+\mu_{v}+c_{v}\right)\left(\lambda+\mu_{b}\right)-c_{v} \beta_{v h} K \mathcal{L}\left\{f_{h}\right\}\left(\lambda+\mu_{h}\right) \mathcal{L}\left\{f_{v}\right\}\left(\lambda+\mu_{v}+c_{v}\right)$,

and $A_{j k}(\lambda)$ is the cofactor of the element $a_{j k}(\lambda)$ of $A(\lambda)$. Note that each $A_{j k}(\lambda)$ is analytic in $\lambda$ for $\operatorname{Re} \lambda \geq 0$.

We have

$$
\begin{aligned}
& \mathcal{L}\left\{\bar{I}_{h}\right\}(\lambda)=\frac{1}{\Delta(\lambda)}\left[A_{11}(\lambda)\left(\bar{I}_{h}(0)+\beta_{v h} \int_{0}^{\infty} e^{-\lambda \bar{t}} \int_{-\infty}^{0} \bar{C}_{v}(\xi) e^{-\mu_{h}(\bar{t}-\xi)} f_{h}(\bar{t}-\xi) d \xi d \bar{t}\right)\right. \\
& \left.+A_{21}(\lambda)\left(\bar{I}_{v}(0)+K \int_{0}^{\infty} e^{-\lambda \bar{t}} \int_{-\infty}^{0} \bar{I}_{h}(\xi) e^{-\left(\mu_{v}+c_{v}\right)(\bar{t}-\xi)} f_{v}(\bar{t}-\xi) d \xi d \bar{t}\right)+A_{31}(\lambda) \bar{C}_{v}(0)\right]
\end{aligned}
$$

Taking the inverse Laplace transform:

$$
\begin{aligned}
& \bar{I}_{h}(t)=\frac{1}{2 \pi i} \int_{\sigma-i \infty}^{\sigma+i \infty} \frac{e^{\lambda t} d \lambda}{\Delta(\lambda)}\left[A_{11}(\lambda)\left(\bar{I}_{h}(0)+\beta_{v h} \int_{0}^{\infty} e^{-\lambda \bar{t}} \int_{-\infty}^{0} \bar{C}_{v}(\xi) e^{-\mu_{h}(\bar{t}-\xi)} f_{h}(\bar{t}-\xi) d \xi d \bar{t}\right)\right. \\
& \left.+A_{21}(\lambda)\left(\bar{I}_{v}(0)+K \int_{0}^{\infty} e^{-\lambda \bar{t}} \int_{-\infty}^{0} \bar{I}_{h}(\xi) e^{-\left(\mu_{v}+c_{v}\right)(\bar{t}-\xi)} f_{v}(\bar{t}-\xi) d \xi d \bar{t}\right)+A_{31}(\lambda) \bar{C}_{v}(0)\right]
\end{aligned}
$$


where the integral is the standard Bromwich integral with the quantity $\sigma$, in the limits, taken as any real number which strictly exceeds the supremum of the real parts of the zeros of $\Delta(\lambda)$. By Cauchy's residue theorem,

$$
\begin{aligned}
& \bar{I}_{h}(t)=\sum_{\{\lambda: \Delta(\lambda)=0\}} \operatorname{Res}\left[\frac { e ^ { \lambda t } } { \Delta ( \lambda ) } \left[A_{11}(\lambda)\left(\bar{I}_{h}(0)+\beta_{v h} \int_{0}^{\infty} e^{-\lambda \bar{t}} \int_{-\infty}^{0} \bar{C}_{v}(\xi) e^{-\mu_{h}(\bar{t}-\xi)} f_{h}(\bar{t}-\xi) d \xi d \bar{t}\right)\right.\right. \\
& \left.\left.+A_{21}(\lambda)\left(\bar{I}_{v}(0)+K \int_{0}^{\infty} e^{-\lambda \bar{t}} \int_{-\infty}^{0} \bar{I}_{h}(\xi) e^{-\left(\mu_{v}+c_{v}\right)(\bar{t}-\xi)} f_{v}(\bar{t}-\xi) d \xi d \bar{t}\right)+A_{31}(\lambda) \bar{C}_{v}(0)\right]\right]
\end{aligned}
$$

where the summation includes the residues at all the poles of the integrand, i.e. all the zeros of $\Delta(\lambda)$. But the equation $\Delta(\lambda)=0$ is the same as the characteristic equation (3.44) that arose in the proof of Theorem 7 , and therefore its roots all satisfy $\operatorname{Re} \lambda<0$. Let $\lambda^{*}$ be the dominant root of $\Delta(\lambda)=0$, so all other roots satisfy $\operatorname{Re} \lambda \leq \lambda^{*}$ and $\lambda^{*}$ itself satisfies $\operatorname{Re} \lambda^{*}<0$. The dominant term in the solution variable $\bar{I}_{h}(t)$ will be attributable to the residue at the dominant root $\lambda=\lambda^{*}$, and therefore

$$
\begin{aligned}
& \bar{I}_{h}(t) \simeq \frac{1}{\Delta^{\prime}\left(\lambda^{*}\right)} e^{\lambda^{*} t}\left[A_{11}\left(\lambda^{*}\right)\left(\bar{I}_{h}(0)+\beta_{v h} \int_{0}^{\infty} e^{-\lambda^{*} \bar{t}} \int_{-\infty}^{0} \bar{C}_{v}(\xi) e^{-\mu_{h}(\bar{t}-\xi)} f_{h}(\bar{t}-\xi) d \xi d \bar{t}\right)\right. \\
& \left.+A_{21}\left(\lambda^{*}\right)\left(\bar{I}_{v}(0)+K \int_{0}^{\infty} e^{-\lambda^{*} \bar{t}} \int_{-\infty}^{0} \bar{I}_{h}(\xi) e^{-\left(\mu_{v}+c_{v}\right)(\bar{t}-\xi)} f_{v}(\bar{t}-\xi) d \xi d \bar{t}\right)+A_{31}\left(\lambda^{*}\right) \bar{C}_{v}(0)\right] \\
& =\frac{1}{\Delta^{\prime}\left(\lambda^{*}\right)}\left[e^{\lambda^{*} t}\left(A_{11}\left(\lambda^{*}\right) \bar{I}_{h}(0)+A_{21}\left(\lambda^{*}\right) \bar{I}_{v}(0)+A_{31}\left(\lambda^{*}\right) \bar{C}_{v}(0)\right)\right. \\
& +A_{11}\left(\lambda^{*}\right) \beta_{v h} \int_{0}^{\infty} e^{\lambda^{*}(t-\bar{t})} \int_{-\infty}^{0} \bar{C}_{v}(\xi) e^{-\mu_{h}(\bar{t}-\xi)} f_{h}(\bar{t}-\xi) d \xi d \bar{t} \\
& \left.+A_{21}\left(\lambda^{*}\right) K \int_{0}^{\infty} e^{\lambda^{*}(t-\bar{t})} \int_{-\infty}^{0} \bar{I}_{h}(\xi) e^{-\left(\mu_{v}+c_{v}\right)(\bar{t}-\xi)} f_{v}(\bar{t}-\xi) d \xi d \bar{t}\right]
\end{aligned}
$$

Note that $\left|e^{\lambda^{*} t}\right|=e^{\left(\operatorname{Re} \lambda^{*}\right) t} \leq 1$ for $t \geq 0$, since $\operatorname{Re} \lambda^{*}<0$. Since $\bar{I}_{h}(\xi), \bar{I}_{v}(\xi), \bar{C}_{v}(\xi) \leq \epsilon$ for all $\xi \leq 0$,

$$
\begin{aligned}
\bar{I}_{h}(t) & =\left|\bar{I}_{h}(t)\right| \leq \frac{\epsilon}{\left|\Delta^{\prime}\left(\lambda^{*}\right)\right|}\left[\left|A_{11}\left(\lambda^{*}\right)\right|+\left|A_{21}\left(\lambda^{*}\right)\right|+\left|A_{31}\left(\lambda^{*}\right)\right|\right. \\
& +\left|A_{11}\left(\lambda^{*}\right)\right| \beta_{v h} \int_{0}^{\infty} e^{\operatorname{Re} \lambda^{*}(t-\bar{t})} \int_{-\infty}^{0} e^{-\mu_{h}(\bar{t}-\xi)} f_{h}(\bar{t}-\xi) d \xi d \bar{t} \\
& \left.+\left|A_{21}\left(\lambda^{*}\right)\right| K \int_{0}^{\infty} e^{\operatorname{Re} \lambda^{*}(t-\bar{t})} \int_{-\infty}^{0} e^{-\left(\mu_{v}+c_{v}\right)(\bar{t}-\xi)} f_{v}(\bar{t}-\xi) d \xi d \bar{t}\right]=: \frac{\epsilon}{\left|\Delta^{\prime}\left(\lambda^{*}\right)\right|} \Theta(t)
\end{aligned}
$$

with $\Theta(t)$ defined as the expression in the large square brackets. By Fatou's lemma, and using that $\operatorname{Re} \lambda^{*}<0$,

$$
\begin{aligned}
\limsup _{t \rightarrow \infty} & \int_{0}^{\infty} e^{\operatorname{Re} \lambda^{*}(t-\bar{t})} \int_{-\infty}^{0} e^{-\mu_{h}(\bar{t}-\xi)} f_{h}(\bar{t}-\xi) d \xi d \bar{t} \\
& \leq \int_{0}^{\infty}\left(\limsup _{t \rightarrow \infty} e^{\operatorname{Re} \lambda^{*}(t-\bar{t})}\right) \int_{-\infty}^{0} e^{-\mu_{h}(\bar{t}-\xi)} f_{h}(\bar{t}-\xi) d \xi d \bar{t}=0
\end{aligned}
$$


and similarly for the other integral. It follows that, as $t \rightarrow \infty, \Theta(t) \rightarrow\left|A_{11}\left(\lambda^{*}\right)\right|+$ $\left|A_{21}\left(\lambda^{*}\right)\right|+\left|A_{31}\left(\lambda^{*}\right)\right|$ and therefore that $\Theta(t)$ is bounded. Moreover, $\Theta(t)$ does not depend on the initial data. The quantity $K$ does depend on $\epsilon$, but $K$ approaches a finite limit as $\epsilon \rightarrow 0$, since $\phi(0)=0$. Thus $\Theta(t)$ is bounded independently of both the initial data and $\epsilon$, for sufficiently small $\epsilon$, and thus we have a bound for $I_{h}(t)$ of the form

$$
I_{h}(t) \leq \bar{I}_{h}(t) \leq k \epsilon
$$

as originally claimed.

\section{Discussion}

The parameter from which we can gain the most useful insight is $R_{0}$, defined by (3.25). According to the predictions of the linearised theory, small introductions of disease will be eradicated if $R_{0}<1$. The quantity $R_{0}$ depends on a number of model parameters and, as is common, it depends more sensitively on some of these parameters than others. Moreover, in practice only some of them are within our control.

The parameter $c_{v}$, the per-capita crushing rate, features four times in expression (3.25) in all, comprising three explicit appearances plus an indirect appearance via $S_{v}^{0}$, which depends on $c_{v}$. Note that $S_{v}^{0}$ decreases as $c_{v}$ increases for realistic choices of the function $b_{v}(\cdot)$. We can have $R_{0}<1$ either by taking $c_{v}$ sufficiently small or sufficiently large. In the former case eradication occurs because it is only crushed lice that transmit LBRF. In the latter case it is because sufficient indiscriminate crushing of all (not just infectious) lice can eradicate LBRF by eradicating the whole lice population. However, the conclusions that can be drawn from these observations are likely to be of limited value. One would assume that there is a wish to eradicate the lice themselves, not just the LBRF they transmit, but eradication of the whole lice population by the sole means of encouraging crushing seems ill advised, since it would effectively also encourage scratching. On the other hand, avoiding crushing eradicates LBRF, because only crushed lice transmit it, but this approach lets the lice live. A strategy of lice control with a minimum of crushing would seem promising, and thus we now turn our attention to the parameter $\mu_{v}$.

Excluding $c_{v}$ it would appear that, of all the parameters we might expect to be able to control, $R_{0}$ is most sensitive to the parameter $\mu_{v}$ which accounts for death of lice (susceptible, exposed and infectious) not attributable to crushing. The parameter $\mu_{v}$ features three times in (3.25) in all, when we recall that $S_{v}^{0}$ depends on $\mu_{v}$. For realistic choices of $b_{v}(\cdot), S_{v}^{0}$ decreases as $\mu_{v}$ increases. Since transmission of LBRF to a human involves the death of the louse, in practice lice need to be present in large numbers to sustain the disease and thus reducing $S_{v}^{0}$ is highly desirable. Also, the Laplace transform term $\hat{f}_{v}\left(\mu_{v}+c_{v}\right)$, in the numerator of (3.25), decreases as $\mu_{v}$ increases. All three influences of $\mu_{v}$ on $R_{0}$ have the effect of decreasing $R_{0}$ as $\mu_{v}$ is increased. The main implication is that we should kill lice without crushing them. It is known that the use of insecticides, general improvements in hygiene, and measures such as discarding infected clothes or bedding (or washing them above $60^{\circ} \mathrm{C}$ ) are effective measures. Note that they will also have the effect of increasing $\mu_{b}$ by removing the infected body fluids of the crushed lice, and an increase in $\mu_{b}$ has a decreasing effect on $R_{0}$ similar (and additional) to the effect on 
$R_{0}$ of an increase in $\mu_{v}$. This ability to substantially reduce $R_{0}$ in simple and inexpensive ways no doubt explains why LBRF is uncommon nowadays.

A further measure that reduces $R_{0}$ is improved access to treatment, although $R_{0}$ is less sensitive to $\nu$ (the per-capita recovery rate from LBRF) than to the parameter $\mu_{v}$ just discussed.

A final implication of the formula (3.25) for $R_{0}$ comes from the presence of the product $\beta_{v h} \beta_{h v}$, which will be proportional to the square of the biting rate. The biting rate is not featured explicitly in our model in this paper but will be one of a product of parameters (including, inter alia, the probability that a bite actually transmits disease) that make up each transmission coefficient (each $\beta$ parameter), so that $R_{0}$ is proportional to the square of the biting rate. This is a standard property of mathematical models of insect-borne diseases and highlights the importance of any measure that reduces biting.

Acknowledgement. We thank the referees for their comments.

\section{References}

[1] BADIAGA, S. \& BROUQUI, P. (2012) Human louse-transmitted infectious diseases. Clinical Microbiology and Infection. 18, 332-337.

[2] Ciervo, A., MANCini, F., di Bernardo, F., GIAMMANCO, A., VitAlE, G., DONES, P., FASCIANA, T., QUARTARO, P., MAZZOLA, G. and REZZA, G. (2016) Louse-borne relapsing fever in young migrants, Sicily, Italy, July-September 2015. Emerging infectious diseases. 22, 152-153.

[3] CUTLER, S.J., ABDISSA, A. \& TRAPE, J.F. (2009) New concepts for the old challenge of African relapsing fever borreliosis. Clinical Microbiology and Infection. 15, 400-406.

[4] GOURLEY, S.A., THIEME, H.R. \& van den DRIESSCHE, P. (2011) Stability and persistence in a model for bluetongue dynamics. SIAM J. Appl. Math. 71, 12801306.

[5] GUBBins, S., CARPENTER, S., BAYLiS, M., WOOD, J.L.N. \& MELLOR, P.S. (2008) Assessing the risk of bluetongue to UK livestock: uncertainty and sensitivity analyses of a temperature-dependent model for the basic reproduction number. $J$. Royal Soc. Interface. 5, 363-371.

[6] HALE, J.K. \& VERDUYN LUNEL, S.M. (1993) Introduction to functionaldifferential equations. Applied Mathematical Sciences, Vol. 99. Springer-Verlag, New York.

[7] HARTEMINK, N.A., PURSE, B.V., MEISWINKEL, R., BROWN, H.E., de KOEIJER, A., ELBERS, A.R., BOENDER, G.-J., ROGERS, D.J. \& HEESTERBEEK, J.A.P. (2009) Mapping the basic reproduction number $\left(R_{0}\right)$ for vector-borne diseases: a case study on bluetongue virus. Epidemics. 1, 153-161. 
[8] HOCH, M., WIESER, A., LÖSCHER, T., MARGOS, G., PÜRNER, F., ZÜHL, J., SEILMAIER, M., BALZER, L., GUGGEMOS, W., RACK-HOCH, A., VON BOTH, U., HAUPTVOGEL, K., SCHÖNBERGER, K., HAUTMANN, W., SING, A. \& FINGERLE, V. (2015) Louse-borne relapsing fever (Borrelia recurrentis) diagnosed in 15 refugees from northeast Africa: epidemiology and preventive control measures, Bavaria, Germany, July to October 2015. Euro Surveill, 20, doi: 10.2807/15607917.ES.2015.20.42.30046.

[9] HORTON, B.J. \& CAREW, A.L. (2015) A comparison of deterministic and stochastic models for predicting the impacts of different sheep body lice (Bovicola ovis) management practices. Animal Production Science. 55, 122-132.

[10] KUANG, Y. (1993) Delay differential equations with applications in population dynamics. Mathematics in Science and Engineering, 191. Academic Press, Inc., Boston, MA.

[11] LAGUNA, M.F. \& RISAU-GUSMAN, S. (2011) Of Lice and Math: Using Models to Understand and Control Populations of Head Lice. PLoS ONE 6(7): e21848.

[12] MERI, T., CUTLER, S.J., BLOM, A.M., MERI, S. \& JOKIRANTA, T.S. (2006) Relapsing fever spirochetes Borrelia recurrentis and B. duttonii acquire complement regulators $\mathrm{C} 4 \mathrm{~b}-$ binding protein and factor $\mathrm{H}$. Infection and immunity. 74, 41574163.

[13] PALMER, C. (2016) The dynamics of vector-borne relapsing diseases. PhD dissertation, University of Montana.

[14] RAOULT, D. \& ROUX, V. (1999) The body louse as a vector of reemerging human diseases. Clinical Infectious Diseases. 29, 888-911.

[15] SMITH, H.L. (1995) Monotone dynamical systems. An introduction to the theory of competitive and cooperative systems. Mathematical Surveys and Monographs, 41. American Mathematical Society, Providence, RI,

[16] SOUTHERN, P.M., Jr. \& SANFORD, J.P. (1969) Relapsing fever: a clinical and microbiological review. Medicine. 48, 129-150. 